\title{
Water chemistry and endangered white-clawed Crayfish: a literature review and field study of water chemistry association in Austropotamobius pallipes
}

\author{
N.R. Haddaway ${ }^{(1),(2), \star}$, R.J.G. Mortimer ${ }^{(3)}$, M. Christmas ${ }^{(4)}$, A.M. Dunn ${ }^{(2)}$
}

Received October 9, 2014

Revised November 11, 2014

Accepted December 2, 2014

Key-words: freshwater, aquatic, conservation, water quality, habitat requirements

\section{ABSTRACT}

Populations of the endangered white-clawed crayfish (Austropotamobius pallipes) have rapidly declined in distribution and density in recent decades as a result of invasive crayfish, disease and habitat degradation. The species is thought to be particularly sensitive to water chemistry, and has been proposed as a bio-indicator of water quality. Here we detail the results of a systematic review of the literature regarding the chemistry of waterbodies inhabited by white-clawed crayfish, along with a wide-scale field study of the chemistry of crayfish-inhabited waterbodies in the UK. We use these data to examine potentially significant variables influencing crayfish distribution. Several variables appear to have thresholds that affect crayfish distribution; crayfish presence was associated with high dissolved oxygen, low conductivity, ammonium, sodium, and phosphate, and to a lesser extent low sulphate, nitrate, and total suspended solids. Some variables (magnesium, potassium, sodium, sulphate, nitrate, and total suspended solids) may be tolerated at moderate to high concentrations in isolation (indicated by the presence of some populations in high levels of these variables), but suites of chemical conditions may act synergistically in situ and must be considered together. Recent efforts to conserve white-clawed crayfish have included relocations to Ark Sites; novel protected habitats with reduced risk of the introduction of disease, invasive crayfish and habitat degradation. We use our findings to propose the first detailed guidelines for common water chemistry variables of potential Ark Sites for the conservation of the species throughout its European range.

\section{RÉSUMÉ}

Chimie de l'eau et écrevisse à pattes blanches en voie de disparition : une revue de la littérature et une étude sur le terrain de la chimie de l'eau associée à Austropotamobius pallipes

Mots-clés : eau douce, aquatique,
Les populations de l'écrevisse à pattes blanches (Austropotamobius pallipes) en voie de disparition, ont rapidement décliné dans leur distribution et leur densité au cours des dernières décennies à cause d'écrevisses invasives, de maladie et de la dégradation de l'habitat. L'espèce est supposée être particulièrement sensible à

(1) Centre for Evidence-Based Conservation, Bangor University, Bangor, LL57 2UW, UK

(2) School of Biology, University of Leeds, LS2 9JT, UK

(3) School of Earth and Environment, University of Leeds, Leeds, LS2 9JT, UK

(4) Environment Agency, Rivers House, 21 Park Square South, Leeds, West Yorkshire, LS1 2QG, UK

*Corresponding author: neal_haddaway@hotmail.com 
conservation, qualité de l'eau, besoins

en habitat la chimie de l'eau, et a été proposée comme un bio-indicateur de la qualité de l'eau. Ici, nous détaillons les résultats d'une revue systématique de la littérature concernant la chimie des masses d'eau habitées par des écrevisses à pattes blanches, avec une étude de terrain à grande échelle de la chimie des masses d'eau abritant des écrevisses au Royaume-Uni. Nous utilisons ces données pour identifier les variables potentiellement importantes qui influent sur la distribution des écrevisses. Plusieurs variables semblent avoir des seuils qui affectent la distribution des écrevisses; la présence d'écrevisses a été associée à de l'oxygène dissous élevé, une faible conductivité, une faible concentration d'ammonium, de sodium et de phosphate, et dans une moindre mesure à une faible concentration en sulfate, nitrate, et matières en suspension. Certaines variables (le magnésium, le potassium, le sodium, le sulfate, le nitrate, et les matières en suspension) peuvent être tolérées à des concentrations modérées à élevées quand il n'y en a qu'une seule en concentration forte (indiqué par la présence de certaines populations à des niveaux élevés de ces variables), mais les conséquences de conditions chimiques peuvent agir en synergie in situ et l'ensemble de ces conséquences doit être considéré. Les récents efforts pour conserver l'écrevisse à pattes blanches comprennent les délocalisations vers des sites Ark; nouveaux habitats protégés avec un risque réduit d'introduction de maladie et d'écrevisses invasives et de dégradation de l'habitat. Nous utilisons nos résultats pour proposer les premières lignes directrices détaillées pour les variables de chimie de l'eau communes de sites Ark potentiels pour la conservation de l'espèce dans son aire européenne.

\section{INTRODUCTION}

The white-clawed crayfish (Austropotamobius pallipes) has a widespread distribution throughout Western Europe, with significant numbers found in Britain (Holdich et al., 1999; Kouba et al., 2014). Despite its wide range, many populations have been lost or dramatically reduced in size in recent decades as a result of crayfish plague, competitive exclusion by invasive non-native crayfish, and habitat degradation (e.g. Gherardi and Holdich, 1999; Holdich et al., 2009). Whilst some populations of $A$. pallipes have been found in relatively low quality water bodies (Holdich et al., 1999), presence of the species is generally reported to be associated with water of 'good' quality; typically, moderately alkaline, low in pollutants, and non-eutrophic waters (e.g. Holdich and Reeve, 1991). The use of $A$. pallipes as a bioindicator species, however, has been debated, since some tolerance to pollutants may exist (see Füreder et al., 2003; Füreder and Reynolds, 2003; Talley and Dagget, 2006).

A. pallipes has been included in the IUCN Red Data List, and is currently listed as endangered (IUCN, 2011). It is also included in Annexes II and V of the European Habitats Directive (Council Directive 92/43/EEC on the Conservation of natural habitats and of wild fauna and flora), with an implicit requirement for the establishment of protected areas for their protection (Special Areas of Conservation), and Appendix 2 of the Bern Convention. Various forms of Environment Agency and Natural England licensing exist in the UK to protect the species from detriment. Conservation efforts to maintain populations of $A$. pallipes in the wild in Britain have recently focussed on relocation of threatened populations. The species is threatened from crayfish plague (Aphanomyces astaci), which has caused the loss of numerous populations, and also from habitat degradation and competition with invasive, non-native crayfish. Animals are removed from areas where risk of infection by crayfish plague, competitive exclusion by invasive non-native crayfish, and population decline due to habitat degradation are high, and placed into habitats that are assessed to have minimal risks from these factors. Such relocation has been termed Ark Site conservation and has undergone substantial public review to produce management guidelines for Ark Site conservation in the UK (Kemp et al., 2000; Peay, 2003, 2009; Whitehouse et al., 2009). However, water chemistry guidelines would be strengthened considerably by a detailed examination of typical water chemistry conditions for the species in its European and British ranges. This has yet to be undertaken 
beyond a regional scale (e.g. Smith et al., 1996; Favaro et al., 2010). Such analysis would allow conservation managers to assess the suitability of potential Ark Sites by relating their water chemistry to the known range of variables from the species' natural range. A degree of suitability can then be attached to these Ark Site water chemistry variables based on their frequency of occurrence in the wild.

Until recently, Austropotamobius pallipes was referred to as a species complex, consisteing of several subspecies in Western Europe. It has now, however, been reclassified as two distinct species (Grandjean et al., 2002); A. pallipes in the British Isles and France, and several subspecies of $A$. italicus in the rest of its range (including Spain and Italy). Since the species are similar in their morphology and habitat requirements and only genetic analysis has shown them to be separate, we include $A$. italicus in this review (clearly stated as a different species where referenced) to make the best use of limited evidence.

Other recent work has been published which aims to link crayfish distributions to the macroinvertebrates on which they feed (e.g. Grandjean et al., 2011; Trouilhé et al., 2012; Jandy et al., 2014). We have chosen to focus instead on water chemistry variables due to the ease with which water samples can be taken and the high level of measurement accuracy attainable. We recognise the merits of studies examining macroinvertebrate prey species distributions with respect to crayfish presence, however.

Herein, we present the findings of an analysis of the evidence regarding water chemistry in sites inhabited by $A$. pallipes in Europe. We also present results of an observational field study of water chemistry in 18 sites inhabited by the species in Britain. We identify variables that may be affecting the distribution of $A$. pallipes and suggest guidelines for the selection of potential Ark Sites for conservation of the species in Britain.

\section{METHODS}

\section{> SYSTEMATIC LITERATURE REVIEW}

Literature searches were carried out using Web of Science (including Web of Science Core Collections, Biosis Previews, MEDLINE, SciELO Citation Index, and Zoological Record) on 13/09/14 for water chemistry associations of $A$. pallipes.

The following search string was used for $A$. pallipes water chemistry associations: ("austropotamobius pallipes" OR white-claw* OR "white claw"” OR whiteclaw*) AND (chemistry OR conductivity OR ammoni* OR nitrate OR nitrite OR phosphate OR chloride OR magnesium OR calcium OR conduct* OR potassium OR sodium OR sulphate OR "dissolved oxygen" OR pH OR TSS OR "total suspended solid"). These water chemistry variables were chosen from an initial assessment of commonly examined variables in the literature. Search results were assessed for relevance in a three-tier approach; title, abstract, and full text. Potentially relevant references were also assessed from within identified articles. Additional relevant articles not found through searches were added using a "snowballing" technique (Jalali and Wohlin, 2012), whereby the reference lists of relevant articles were scanned for further relevant studies.

In order to produce values with which to compare waters inhabited by $A$. pallipes, mean values reported for major global and European rivers were collated from the literature. The following water chemistry variables for global and European rivers were obtained from Berner and Berner (1996); calcium, magnesium, sodium, potassium, chloride, and sulphate. Values for conductivity, dissolved oxygen, ammonium, nitrate, total dissolved solids, $\mathrm{pH}$, and phosphate were obtained from an independent review of the literature on 24/08/2012. Twenty-two additional articles (see Appendix 1 for details) were identified from a search of the literature on global and European rivers using Web of Science. These articles were reviewed and data on the water chemistry variables described above were extracted to generate means and ranges. In addition, representative values for rivers in the United Kingdom were obtained from the Harmonised Monitoring Scheme, a Defra and CEH initiative to monitor water chemistry. 15 regions across England and Wales were chosen as representative regions also currently/previously inhabited by $A$. pallipes (see Appendix 2 for details of data and their sources). 
Table I

Sites inhabited by white-clawed crayfish (A. pallipes) that were sampled for water chemistry.

\begin{tabular}{|c|c|c|c|c|}
\hline Site & Location & $\begin{array}{l}\text { Latitude } \\
\text { (WGS84) }\end{array}$ & $\begin{array}{c}\text { Longitude } \\
\text { (WGS84) }\end{array}$ & Tyрe \\
\hline Adel Beck & Leeds, West Yorkshire & $52^{\circ} 42^{\prime} 33.47^{\prime \prime} \mathrm{N}$ & $2^{\circ} 27^{\prime} 58.01^{\prime \prime} \mathrm{W}$ & Lotic \\
\hline Brasside Pond & Durham, County Durham & $54^{\circ} 48^{\prime} 29.10^{\prime \prime} \mathrm{N}$ & $1^{\circ} 33^{\prime} 00.91^{\prime \prime} \mathrm{W}$ & Lentic \\
\hline Broomlee Lough & Hexham, Northumberland & $55^{\circ} 01^{\prime} 18.48^{\prime \prime} \mathrm{N}$ & $2^{\circ} 19^{\prime} 49.34^{\prime \prime} \mathrm{W}$ & Lentic \\
\hline Coppice Pond & Bingley, West Yorkshire & $53^{\circ} 50^{\prime} 47.06^{\prime \prime} \mathrm{N}$ & $1^{\circ} 51^{\prime} 54.77^{\prime \prime} \mathrm{W}$ & Lentic \\
\hline Cound Brook & Condover, Shropshire & $52^{\circ} 39^{\prime} 24.17^{\prime \prime} \mathrm{N}$ & $2^{\circ} 43^{\prime} 00.22^{\prime \prime} W$ & Lotic \\
\hline Crook Burn & Hexham, Northumberland & $55^{\circ} 02^{\prime} 06.98^{\prime \prime} \mathrm{N}$ & $2^{\circ} 17^{\prime} 53.71^{\prime \prime} \mathrm{W}$ & Lotic \\
\hline Dean Brook & Huddersfield, West Yorkshire & $53^{\circ} 37^{\prime} 04.53^{\prime \prime} \mathrm{N}$ & $1^{\circ} 47^{\prime} 55.76^{\prime \prime} \mathrm{W}$ & Lotic \\
\hline Halleypike Lough & Hexham, Northumberland & $55^{\circ} 02^{\prime} 28.66^{\prime \prime} \mathrm{N}$ & $2^{\circ} 17^{\prime} 50.49^{\prime \prime} W$ & Lentic \\
\hline Meanwood Beck & Leeds, West Yorkshire & $53^{\circ} 52^{\prime} 18.71^{\prime \prime} \mathrm{N}$ & $1^{\circ} 37^{\prime} 21.75^{\prime \prime} \mathrm{W}$ & Lotic \\
\hline Pinfold Dam & Huddersfield, West Yorkshire & $53^{\circ} 38^{\prime} 18.09^{\prime \prime} \mathrm{N}$ & $1^{\circ} 49^{\prime} 02.92^{\prime \prime} \mathrm{W}$ & Lentic \\
\hline River Derwent & Scarborough, North Yorkshire & $54^{\circ} 11^{\prime} 50.63^{\prime \prime} \mathrm{N}$ & $0^{\circ} 30^{\prime} 08.29^{\prime \prime} \mathrm{W}$ & Lotic \\
\hline River Kent & Kendal, Cumbria & $54^{\circ} 19^{\prime} 46.69^{\prime \prime} \mathrm{N}$ & $2^{\circ} 45^{\prime} 09.32^{\prime \prime} \mathrm{W}$ & Lotic \\
\hline River Redlake & New Invention, Shropshire & $52^{\circ} 23^{\prime} 07.86^{\prime \prime} \mathrm{N}$ & $3^{\circ} 02^{\prime} 56.20^{\prime \prime} \mathrm{W}$ & Lotic \\
\hline River Wansbeck & Rothley, Northumberland & $55^{\circ} 08^{\prime} 54.89^{\prime \prime} \mathrm{N}$ & $1^{\circ} 56^{\prime} 53.18^{\prime \prime} \mathrm{W}$ & Lotic \\
\hline Robsheugh Burn & Milbourne, Northumberland & $55^{\circ} 03^{\prime} 46.96^{\prime \prime} \mathrm{N}$ & $1^{\circ} 50^{\prime} 59.05^{\prime \prime} \mathrm{W}$ & Lotic \\
\hline Simpson's Pool & Horsehay, Shropshire & $52^{\circ} 39^{\prime} 48.06^{\prime \prime} \mathrm{N}$ & $2^{\circ} 29^{\prime} 26.74^{\prime \prime} \mathrm{W}$ & Lentic \\
\hline Trench Pool & Telford, Shropshire & $52^{\circ} 42^{\prime} 33.47^{\prime \prime} \mathrm{N}$ & $2^{\circ} 27^{\prime} 58.01^{\prime \prime} W$ & Lentic \\
\hline Wyke Beck & Leeds, West Yorkshire & $53^{\circ} 46^{\prime} 58.85^{\prime \prime} \mathrm{N}$ & $1^{\circ} 29^{\prime} 19.45^{\prime \prime} \mathrm{W}$ & Lotic \\
\hline
\end{tabular}

Reported water chemistry variables have been converted to $\mathrm{mg} \cdot \mathrm{L}^{-1}$, with the exception of conductivity $\left(\mu \mathrm{S} \cdot \mathrm{cm}^{-1}\right)$. Most authors have provided a mean, and a maximum and minimum (range) for the rivers examined, whilst some have presented only means or ranges. Graphed global, European and UK values are presented as ranges of means (mean mean, minimum mean, and maximum mean), rather than ranges in absolute values. This method allows discriminatory ability between these values and those for crayfish-inhabited waters. Since global and European values presented are ranges of means, some concentrations for crayfish-inhabited waters may therefore exceed these ranges.

\section{> WATER CHEMISTRY ANALYSIS OF UK POPULATIONS}

Water chemistry was recorded at 18 sites known to be inhabited by $A$. pallipes (see Table I) throughout the Midlands and North England between May and October 2009. Potassium, magnesium, calcium, sodium, chloride, nitrate, sulphate, and phosphate were measured from water samples analysed at the University of Leeds. Dissolved oxygen was measured on-site. Samples of filtered $(0.45 \mu \mathrm{m})$ water were frozen and run through a Dionex ion chromatograph (ICS-90 machine) and gas diffusion flow injection analysis on FIA (ammonium only). Integration was then examined for each sample, and peaks adjusted if necessary to increase accuracy.

Without detailed long-term study, it is impossible to identify whether the absence of $A$. pallipes from a water body is due to local extinction or to historical absence. For this reason, presence/absence comparisons were not carried out in this study. Descriptive statistics (means and ranges) were produced and used to compare with European and global means in order to identify potential patterns in $A$. pallipes-inhabited waters.

\section{RESULTS}

Searches in Web of Science using the crayfish and water chemistry search string resulted in 155 hits. Thirty-two results remained after title- and abstract- level screening, and 23 following full text screening. A total of 23 articles were found to present water chemistry data for at least one variable in waters inhabited by $A$. pallipes across Europe (Wales, England, 

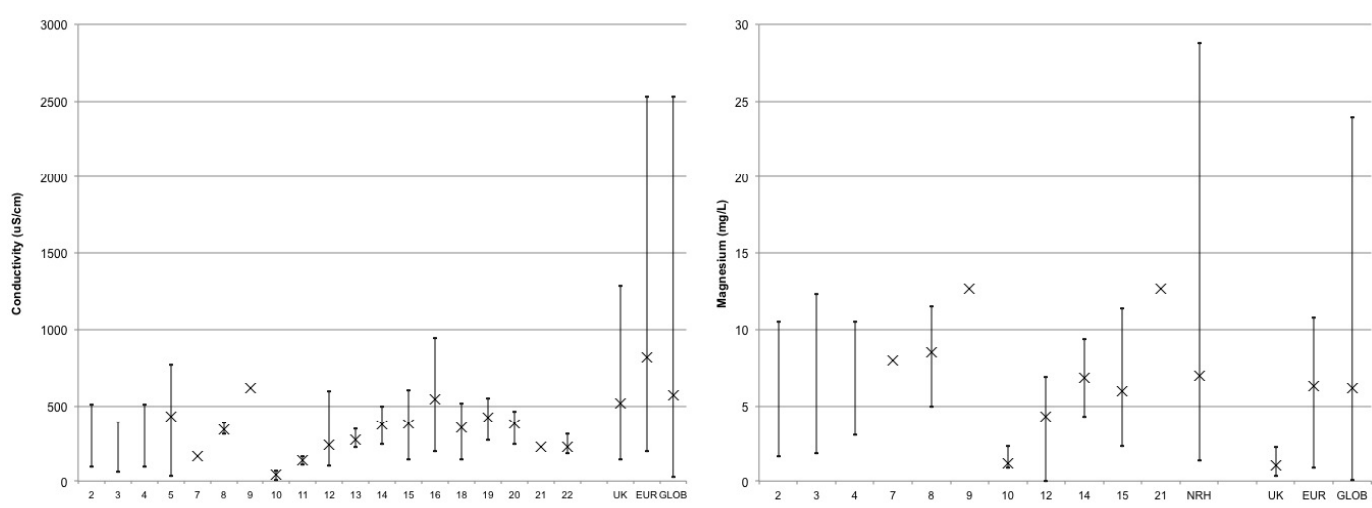

a) Conductivity

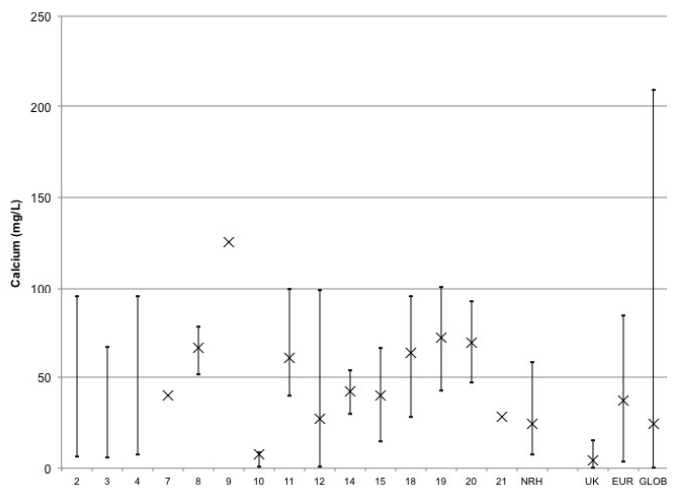

b) Magnesium

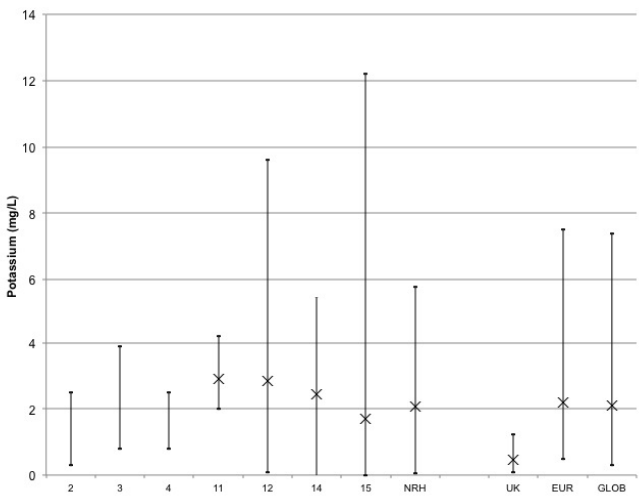

c) Calcium

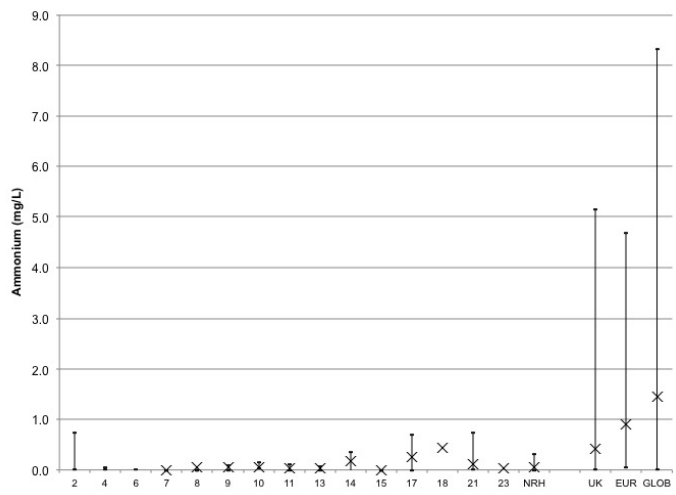

d) Potassium

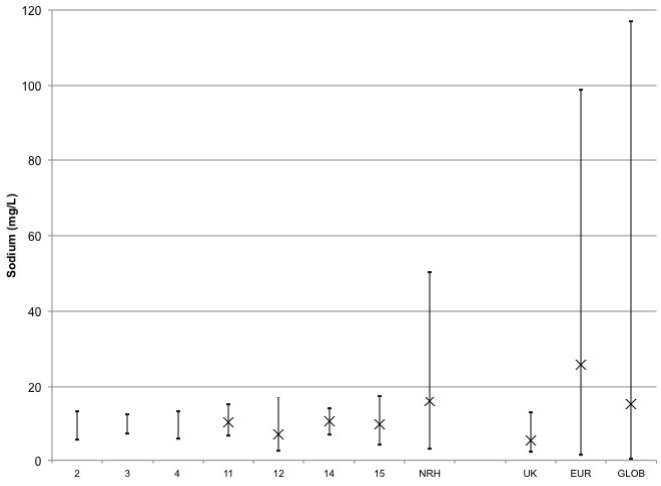

e) Ammonium

f) Sodium

\section{Figure 1}

a-f. Cited levels (mean and range) of major water chemistry variables in waters inhabited by A. pallipes, along with means for European (ER) and worldwide (WR) rivers obtained from Berner and Berner (1996) and an independent review of the literature. See Figure $1 \mathrm{~m}$ for author codes.

Ireland, France, Germany, Spain, Italy, Bosnia and Herzegovina and Croatia). Three studies referred to the species Austropotamobius italicus, which was reclassified from Austropotamobius pallipes italicus (Grandjean et al., 2002). These studies have been included here because of the species' significant phylogenetic similarities (Grandjean et al., 2002). One article modelled water chemistry variables in waters inhabited by $A$. pallipes but did not present the data or summary statistics and could therefore not be included (Favaro et al., 2011). Means and/or maxima and minima were extracted from sources and are presented in Figure 1 (see Appendix 3 for summary data used in these figures). 


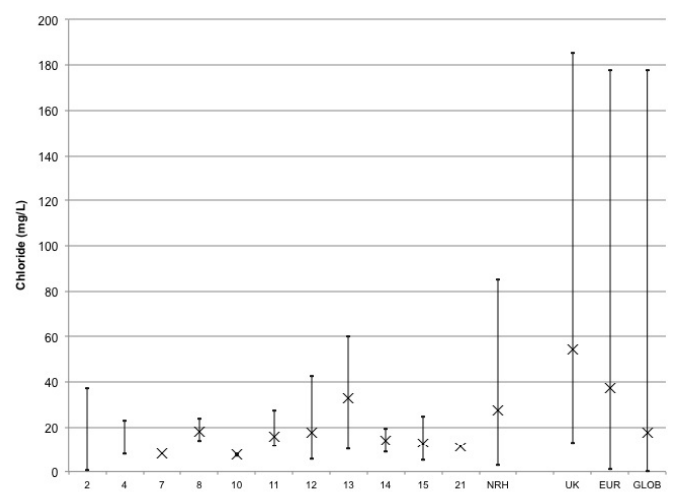

g) Chloride

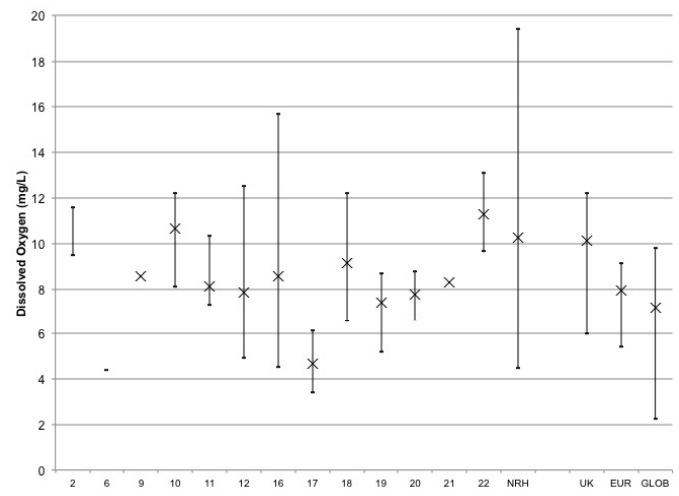

i) Dissolved Oxygen

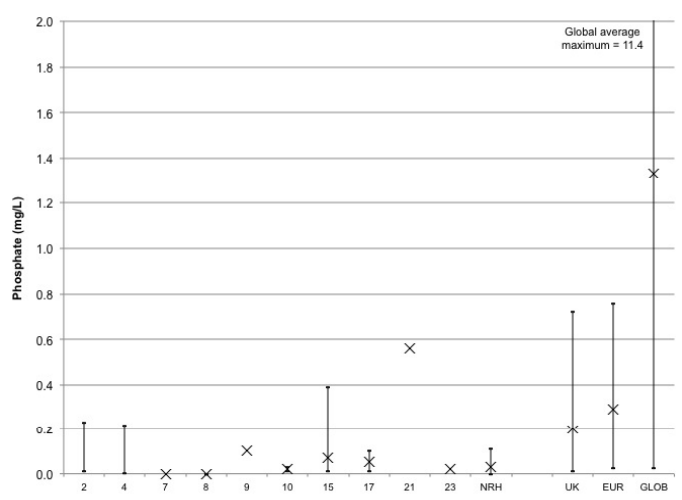

k) Phosphate

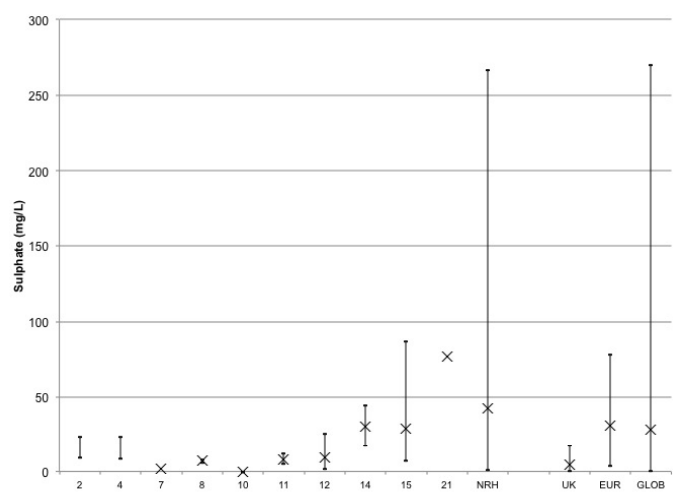

h) Sulphate

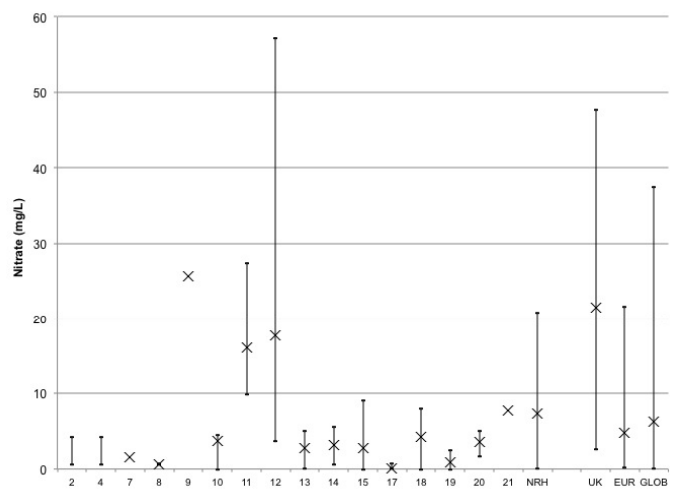

j) Nitrate

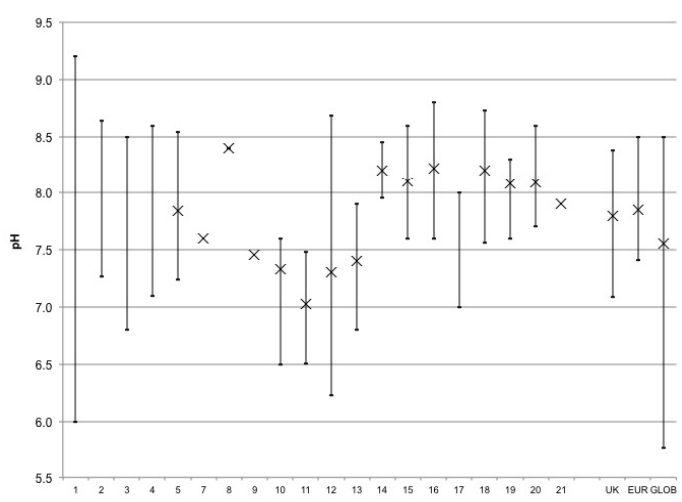

I) $\mathrm{pH}$

Figure 1

Continued. g-l. Cited levels (mean and range) of major water chemistry variables in waters inhabited by A. pallipes, along with means for European (ER) and worldwide (WR) rivers obtained from Berner and Berner (1996) and an independent review of the literature. See Figure $1 \mathrm{~m}$ for author codes.

The data from the review of water chemistry for waters inhabited by $A$. pallipes are presented in Figure 1 and results of the water chemistry analysis of UK waters are presented in Table II.

\section{> CONDUCTIVITY}

All populations of $A$. pallipes reported in the literature lie in the lower range of conductivity reported for UK, global and European rivers (Figure 1a). Waters with conductivity above $700 \mu \mathrm{S} \cdot \mathrm{cm}^{-1}$ are typically polluted or brackish/saline. Two studies reported levels in excess of 


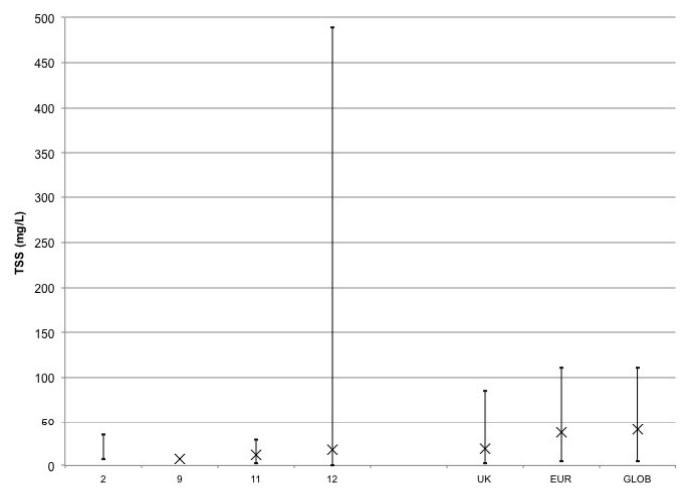

m) Total Suspended Solids (TSS)

\begin{tabular}{clcll}
\hline Code & Author & Year & Country & Notes \\
\hline 1 & Jay and Holdich & 1981 & Wales & \\
2 & Foster & 1995 & Wales & \\
3 & Lilley et al. & 1979 & England & \\
4 & Smith et al. & 1996 & England & \\
5 & Demers and Reynolds & 2002 & Ireland & \\
6 & Lyons and Kelly-Quinn & 2003 & Ireland & \\
7 & Arrignon and Roché & 1983 & France & \\
8 & Arrignon et al. & 1993 & France & \\
9 & Broquet et al. & 2002 & France & \\
10 & Reyjol and Roquelpo & 2002 & France & \\
11 & Trouilhé et al. & 2003 & France & \\
12 & Trouilhé et al. & 2007 & France & \\
13 & Troschel & 1997 & Germany & Standard deviations \\
& Garcia-Arberas and Rallo & 2000 & Spain & instead of min/max \\
14 & Gan & 2002 & Spain & \\
15 & Rallo and Garcia-Arberas & 2005 & Italy & Austropotamobius italicus \\
16 & Nardi et al. & 2005 & Italy & \\
17 & Scalici and Gibertini & 2006 & Italy & \\
18 & Renai et al. & 2007 & Italy & \\
19 & Barbaresi et al. & 2008 & Italy & Austropotamobius italicus \\
20 & Brusconi et al. & 2010 & Italy & \\
21 & Favaro et al. & & Bosnia and & \\
22 & Trožic -Borovac & 2011 & Herzegovina & \\
23 & Gottstein & 1999 & Croatia & Austropotamobius italicus \\
NRH & This study & 2009 & England &
\end{tabular}

\section{Figure 1}

Continued. $m$. Cited levels (mean and range) of major total suspended solids (TSS) in waters inhabited by A. pallipes, along with means for European (ER) and worldwide (WR) rivers obtained from Berner and Berner (1996) and an independent review of the literature. Author codes, years, and locations presented for preceding graphs.

this threshold; Demers and Reynolds (2002) in Ireland, and Nardi et al. (2005) in Italy (although, this was $A$. italicus).

\section{> MAGNESIUM $\left[\mathrm{Mg}^{2+}\right]$}

The wide range of values of magnesium concentration in rivers inhabited by the white-clawed crayfish suggests little association with waters of specific magnesium concentration (i.e. high or low) (Figure 1b). For example, Broquet et al. (2002), Smith et al. (1996), and Favaro et al. (2010) found populations in waters of higher magnesium concentration than the ranges reported for European river means. These higher values lie within the range of global means for magnesium concentration, however. The results from our field survey concur with this lack of association, with minimum and mean values falling well within cited levels and major European river ranges. One site, Trench Pool, had extremely high magnesium $\left(28.8 \mathrm{mg} \cdot \mathrm{L}^{-1}\right)$. This site is known to suffer from urban pollution, despite possessing a substantial population of $A$. pallipes (pers. obs.). The site is an actively used fishing reservoir in an urban area of Trench, Shropshire. An old noticeboard at the entrance to the site warns users of the risks of bluegreen algal blooms, but it is unknown whether this is a current or historic concern. However, other sites did not exceed $12 \mathrm{mg} \cdot \mathrm{L}^{-1}$.

\section{$>$ CALCIUM $\left[\mathrm{Ca}^{2+}\right]$}

A. pallipes populations have been found across the range of European means of calcium concentration. Some authors suggested that the species is associated with concentrations in the lower range of European means (Rallo and Garcia-Arberas, 2002; Reyjol and Roqueplo, 2002). Others, for example Broquet et al. (2002), noted crayfish in higher concentrations than the range of European means, but with values that fell within the range of global means.

It has been suggested that a lower limit of $5 \mathrm{mg} \cdot \mathrm{L}^{-1}$ calcium concentration restricts the presence of $A$. pallipes (Greenaway, 1974), as supported by observations of Jay and Holdich (1981) and Smith et al. (1996) in the British Isles, and Trouilhé et al. (2007) in France. Furthermore, laboratory manipulations involving Astacus astacus by Rukke (2002) showed stunted 


\begin{tabular}{|c|c|c|c|c|c|c|c|c|c|c|c|c|c|c|c|c|c|c|c|}
\hline 8 & $\begin{array}{l}1 \\
\vdots \\
\vdots \\
\vdots\end{array} \mid \begin{array}{l}\infty \\
b \\
\infty\end{array}$ & & & & & 命. & & लै & & $\mid \begin{array}{l}9 \\
\text { o } \\
\infty\end{array}$ & $\begin{array}{l}\text { 웅 } \\
\text { ஸे }\end{array}$ & 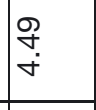 & $\begin{array}{l}\hat{\sigma} \\
\dot{\sigma}\end{array}$ & $\begin{array}{l}8 \\
\ddot{\circ} \\
\oplus\end{array}$ & & \begin{tabular}{l}
\multirow{O}{S}{} \\
$\stackrel{\text { }}{\sim}$
\end{tabular} & $\begin{array}{l}\stackrel{0}{\infty} \\
\stackrel{1}{\infty}\end{array}$ & & in \\
\hline$\frac{7}{\frac{3}{0}}$ & (1) & 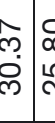 & & 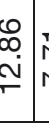 & $\stackrel{\Gamma}{\sim}$ & $\begin{array}{l}\bar{\sigma} \\
\dot{0}\end{array}$ & $\begin{array}{l}\stackrel{D}{N} \\
\stackrel{N}{N}\end{array} \mid$ & 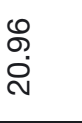 & लె. & $\mid \begin{array}{l}0 \\
\infty \\
\dot{m} \\
\dot{m}\end{array}$ & $\begin{array}{l}\infty \\
\infty \\
\\
\text { Na }\end{array}$ & $\mid \begin{array}{l}R \\
0 \\
\text { en }\end{array}$ & 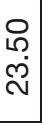 & $\mid$\begin{tabular}{l}
0 \\
0 \\
0 \\
\hdashline
\end{tabular} & 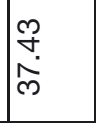 & 竎 & $\begin{array}{l}\infty \\
\infty \\
0 \\
-1\end{array}$ & $\left|\begin{array}{l}\bar{m} \\
\infty \\
\infty \\
1\end{array}\right|$ & స̃ \\
\hline $\begin{array}{l}5 \\
0 \\
0 \\
0 \\
\frac{10}{20} \\
\frac{10}{2}\end{array}$ & \begin{tabular}{l|l}
$\overrightarrow{1}$ \\
$\dot{2}$ \\
$\dot{2}$
\end{tabular} & & & & & 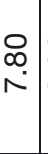 & $\begin{array}{c}\widetilde{N} \\
\stackrel{\sim}{\sim}\end{array}$ & $\begin{array}{l}\mathscr{8} \\
\ddot{\sigma}\end{array}$ & $\stackrel{8}{\square}$ & & $\mid \begin{array}{l}0 \\
\infty \\
\infty\end{array}$ & 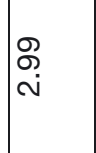 & $\begin{array}{l}\infty \\
0 \\
\dot{m}\end{array}$ & | & $\frac{R}{R}$ & 茂 & $\mid \begin{array}{l}0 \\
\infty \\
\infty\end{array}$ & & 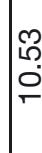 \\
\hline \begin{tabular}{|l|}
$\frac{3}{4}$ \\
0 \\
0 \\
0 \\
0 \\
0 \\
\end{tabular} & ‘े & & S & & & $\begin{array}{l}\mathscr{D} \\
\stackrel{\sim}{*}\end{array} \mid$ & $\frac{10}{0}$ & $\stackrel{\text { }}{\stackrel{N}{N}}$ & ְె. & 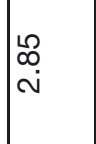 & în & $\stackrel{\bar{\lambda}}{\mathrm{N}}$ & $\begin{array}{l}\stackrel{s}{0} \\
+\end{array}$ & 足 & $\stackrel{+}{0}$ & $\begin{array}{l}\infty \\
\infty \\
\infty \\
\end{array}$ & $\begin{array}{l}\infty \\
\infty \\
\sim \\
\sim\end{array}$ & $\left|\begin{array}{c}\infty \\
1 \\
i \\
\omega^{\circ}\end{array}\right|$ & 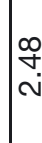 \\
\hline \begin{tabular}{l|l} 
& \\
\\
\\
\end{tabular} & 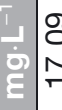 & \begin{tabular}{l|l}
8 & 0 \\
0 & 1 \\
\hdashline & 7 \\
\hdashline & 7
\end{tabular} & & 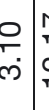 & 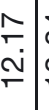 & $\begin{array}{l}\tilde{m} \\
\stackrel{m}{\sigma} \\
\end{array}$ & \begin{tabular}{l}
$\mathscr{L}$ \\
\hdashline \\
$\dot{\sigma}$
\end{tabular} \mid & $\begin{array}{l}\infty \\
\infty \\
\text { Na } \\
\text { and }\end{array}$ & 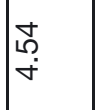 & & 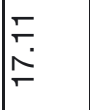 & $\begin{array}{l}\bar{\sigma} \\
\bar{m}\end{array}$ & 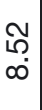 & $\stackrel{0}{\stackrel{0}{r}}$ & $\begin{array}{l}\infty \\
0 \\
0 \\
0 \\
0\end{array}$ & 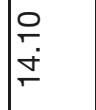 & ס & $\begin{array}{l}0 \\
m \\
0 \\
0 \\
10\end{array}$ & in \\
\hline \begin{tabular}{l|}
3 \\
\\
\\
\\
\\
\\
\end{tabular} & 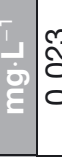 & 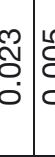 & & & $\begin{array}{l}m \\
\\
0 \\
0\end{array}$ & $\begin{array}{l}\stackrel{n}{n} \\
0 \\
0 \\
0\end{array}$ & $\begin{array}{l}0 \\
\infty \\
0 \\
0 \\
0\end{array}$ & $\begin{array}{l}\infty \\
\tilde{ల} \\
0 \\
0\end{array}$ & 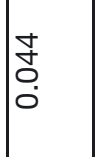 & ס్ర & $\mid \begin{array}{l}\mathscr{O} \\
0 \\
0 \\
0\end{array}$ & 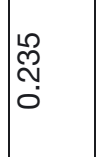 & $\begin{array}{l}0 \\
\tilde{ల} \\
0 \\
0 \\
0\end{array}$ & స̂. & 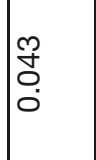 & 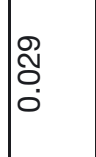 & 足 & & 足 \\
\hline 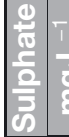 & 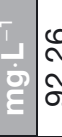 & 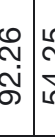 & & 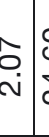 & $\begin{array}{l}\stackrel{0}{0} \\
\dot{\sim}\end{array}$ & $\begin{array}{l}\dot{N} \\
\dot{\mathrm{N}}\end{array}$ & 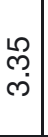 & $\begin{array}{l}\text { \& } \\
\dot{q} \\
\dot{q}\end{array}$ & 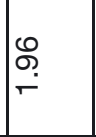 & $\begin{array}{l}\infty \\
0 \\
\delta \\
0 \\
0\end{array}$ & 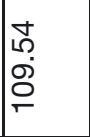 & $\begin{array}{l}\infty \\
\infty \\
\infty \\
\infty \\
m\end{array}$ & $\begin{array}{l}\underset{\tau}{\tau} \\
\stackrel{\sim}{\sim}\end{array}$ & î. & 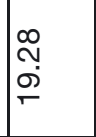 & $\begin{array}{l}\mathscr{O} \\
\dot{f} \\
\dot{f}\end{array}$ & $\begin{array}{l}\infty \\
\infty \\
0 \\
0\end{array}$ & 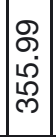 & \begin{tabular}{l}
$\infty$ \\
\multirow{\gamma}{*}{} \\
\multirow{\gamma}{*}{}
\end{tabular} \\
\hline 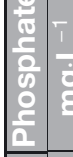 & ڤ) & $\begin{array}{c}\text { ले } \\
\text { ò } \\
\text { ò }\end{array}$ & & & $\begin{array}{l}8 \\
8 \\
0 \\
0\end{array}$ & $\begin{array}{l}0 \\
\overline{0} \\
\\
\end{array}$ & $\begin{array}{l}8 \\
0 \\
0 \\
0\end{array}$ & $\begin{array}{l}\text { ח } \\
\infty \\
0 \\
0 \\
0\end{array}$ & ס्. & $\mid \begin{array}{l}0 \\
0 \\
0 \\
0\end{array}$ & ${ }_{0}^{\infty}$ & $\mid$\begin{tabular}{l}
0 \\
\multirow{O}{0}{} \\
0 \\
0
\end{tabular} & $\begin{array}{l}\tilde{N} \\
0 \\
0 \\
0\end{array}$ & $\begin{array}{l}\infty \\
\tilde{\mathscr{D}} \\
0 \\
0\end{array}$ & $\mid \begin{array}{l}\text { P } \\
\text { o } \\
\text { o. }\end{array}$ & 令 & $\mid \begin{array}{l}\text { L } \\
0 \\
0\end{array}$ & & $\begin{array}{l}1 \\
0 \\
0\end{array}$ \\
\hline$\frac{9}{20}$ & 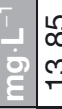 & $\begin{array}{l}\infty \\
\infty \\
\infty \\
\\
\end{array}$ & ॄே. & 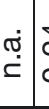 & : & $\begin{array}{l}\hat{N} \\
\dot{\nabla} \\
+\end{array}$ & \begin{tabular}{l}
0 \\
$\bullet$ \\
\hdashline \\
\hdashline
\end{tabular} \mid & $\begin{array}{l}\mathcal{N} \\
\infty \\
-\infty\end{array}$ & $\check{I}_{0}$ & 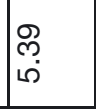 & $\begin{array}{l}\mathscr{M} \\
\stackrel{0}{0} \\
\stackrel{0}{ }\end{array}$ & 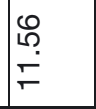 & $\frac{0}{6}$ & 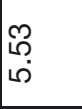 & | & $\begin{array}{l}\text { lo } \\
\text { ले }\end{array}$ & ஷ্ণ் & & $\mid \begin{array}{l}\bar{\sigma} \\
\overline{1}\end{array}$ \\
\hline 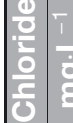 & 竞 & 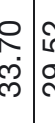 & 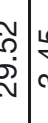 & 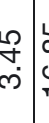 & 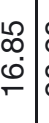 & 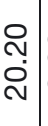 & \begin{tabular}{|l|}
$\infty$ \\
$o$ \\
\end{tabular} \mid & 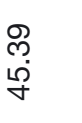 & 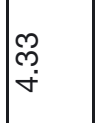 & 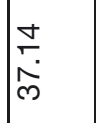 & $\begin{array}{l}\mathscr{8} \\
\stackrel{2}{\text { D }}\end{array}$ & 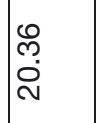 & 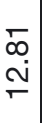 & 离 & 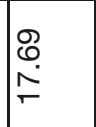 & $\begin{array}{l}\infty \\
\infty \\
\stackrel{\infty}{N} \\
\end{array}$ & 电 & & $\begin{array}{l}\bar{\infty} \\
\dot{\sigma}\end{array}$ \\
\hline & 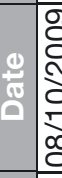 & 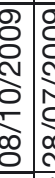 & & 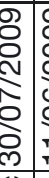 & 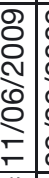 & 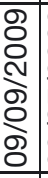 & 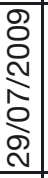 & 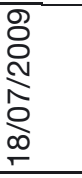 & 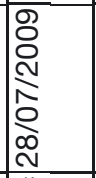 & 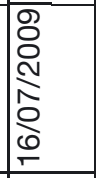 & 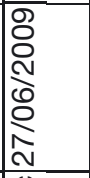 & 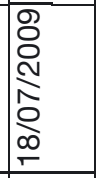 & 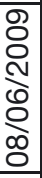 & 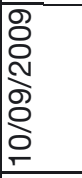 & 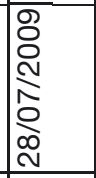 & 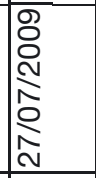 & 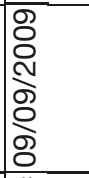 & 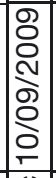 & 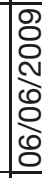 \\
\hline & 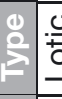 & 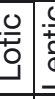 & & 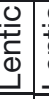 & 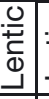 & .0. & $\begin{array}{l}0 \\
\\
\end{array}$ & 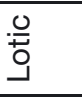 & 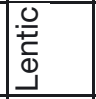 & 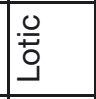 & 吕 & 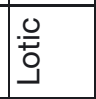 & .0. & 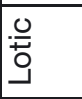 & 菩 & 䓫 & 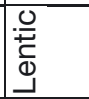 & & 음 \\
\hline & 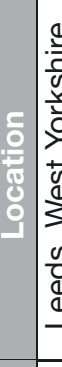 & 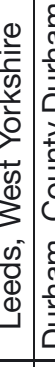 & 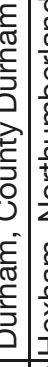 & 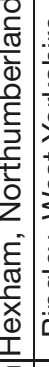 & 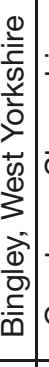 & 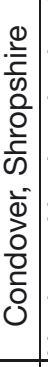 & 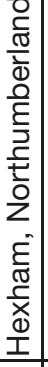 & 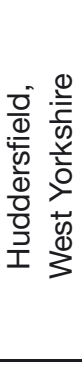 & 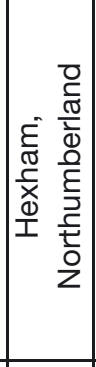 & 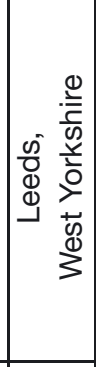 & 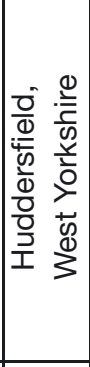 & 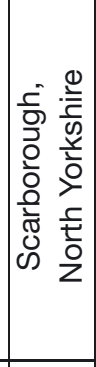 & 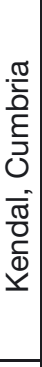 & 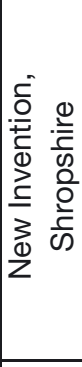 & 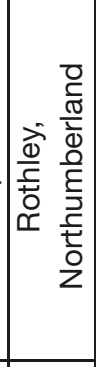 & 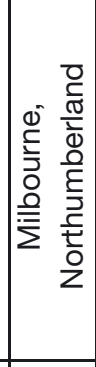 & 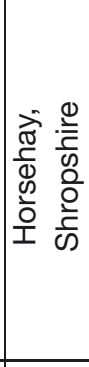 & 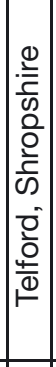 & 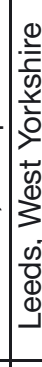 \\
\hline & 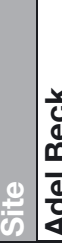 & 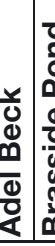 & 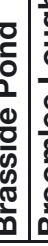 & 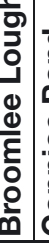 & 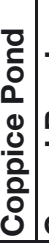 & 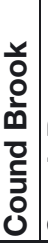 & 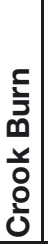 & 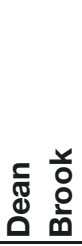 & 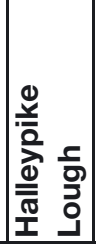 & 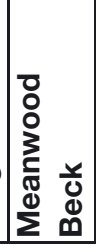 & 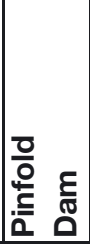 & 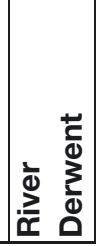 & 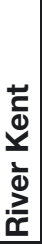 & 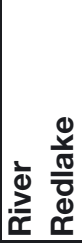 & 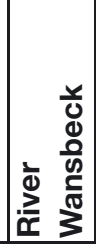 & 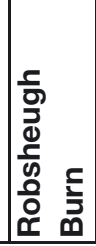 & 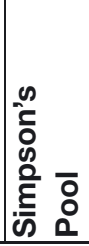 & 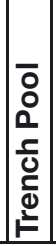 & $\begin{array}{l}y \\
0 \\
0 \\
0 \\
0 \\
0 \\
y \\
z \\
z\end{array}$ \\
\hline
\end{tabular}


growth and reduced survival below $5 \mathrm{mg} \cdot \mathrm{L}^{-1}$ calcium compared to that at $10 \mathrm{mg} \cdot \mathrm{L}^{-1}$. However, one study found populations inhabiting rivers with calcium concentrations below $2.5 \mathrm{mg} \cdot \mathrm{L}^{-1}$ (Reyjol and Roqueplo, 2002) and one as low as $1 \mathrm{mg} \cdot \mathrm{L}^{-1}$ (Trouilhé et al., 2003). Crayfish can, therefore, evidently survive below the proposed $5 \mathrm{mg} \cdot \mathrm{L}^{-1}$ threshold. However, the majority of populations have been found at calcium concentrations greater than $5 \mathrm{mg} \cdot \mathrm{L}^{-1}$ (Figure 1c) and lie close to the range of cited means for European rivers. Our results also show little evidence of association, being similar to major European rivers and many of the cited studies. The minimum calcium concentration found in the UK $A$. pallipes-inhabited sites in our study was $7.4 \mathrm{mg} \cdot \mathrm{L}^{-1}$, in accord with a lower limit of $5 \mathrm{mg} \cdot \mathrm{L}^{-1}$.

\section{> POTASSIUM $\left[K^{+}\right]$}

Populations of $A$. pallipes have been found in water with potassium concentrations similar to those of mean global and European waters (Figure 1d) with two studies finding populations associated with concentrations in the lower ranges of global and European means (Rallo and Garcia-Arberas, 2002; Trouilhé et al., 2007). Given the wide range of potassium concentrations found in waters inhabited by $A$. pallipes, no association for specific potassium concentrations can be identified. Similarly, our field results found no evidence for an association of $A$. pallipes with specific potassium concentration, and resemble both major European and global rivers.

\section{$>$ AMMONIUM $\left[\mathrm{NH}_{4}^{+}\right]$}

A. pallipes populations have all been found in rivers containing low concentrations of ammonium relative to cited means for UK, European and global rivers (Figure 1e), although three studies reported much higher ranges than the others (Foster, 1995; Rallo and Garcia-Arberas, 2002; Favaro et al., 2010). This may indicate an intolerance to high ammonium levels in accordance with known toxicity of the ion to aquatic organisms. However, the toxicity study of Meade and Watts (1995) suggests that crayfish may not be so sensitive to ammonium alone, but to the combined effects of ammonium and nitrate or nitrite. Our field study also found ammonium concentrations in crayfish-inhabited waters to lie in the lower range of UK, European and global rivers. Our mean ammonium concentration was highly influenced by one site, the River Derwent, with relatively elevated ammonium. However, this site was suffering from seasonal flooding, with rotting leaf litter the likely cause of raised ammonium concentrations (Baldy et al., 2007).

\section{$>$ SODIUM $\left[\mathrm{Na}^{+}\right]$}

Populations of $A$. pallipes reported in the literate have only been found in the lower range values for European and global river means for sodium, as shown in Figure 1f. This may indicate a low tolerance for elevated sodium concentrations. However, UK river sodium levels reported by the Harmonised Monitoring Scheme are very similar to those reported for crayfish in the literature. Surprisingly, our field study found levels of sodium at 5/18 of the sites to be higher than previously cited levels for A. pallipes, and one site, Trench Pool, had levels well above the mean values for major European and global rivers.

\section{$>$ CHLORIDE $\left[\mathrm{Cl}^{-}\right]$}

Figure $1 \mathrm{~g}$ shows that reported chloride concentrations of waters inhabited by $A$. pallipes in the literature lie around the average cited European and global river means, with no populations found in higher concentrations than $60 \mathrm{mg} \cdot \mathrm{L}^{-1}$. No clear patterns in association are obvious, 
other than a lack of populations in high chloride concentrations typical of polluted water. Our field results concur with this finding despite some populations occurring in concentrations above other published studies. Nevertheless, we found crayfish populations in waters with low chloride concentration relative to UK, European and global rivers.

\section{$>$ SULPHATE $\left[\mathrm{SO}_{4}^{2-}\right]$}

The majority of $A$. pallipes populations have been found inhabiting waters with sulphate concentrations lower than those reported for major European rivers (Figure 1h), with two notable exceptions; in Italy (Favaro et al., 2010) and Spain (Rallo and Garcia-Arberas, 2002). Other studies appear to have found an association of the species with low concentrations of sulphate relative to European and global rivers, however (but similar to values reported in the Harmonised Monitoring Scheme). This suggests an association with low levels, but tolerance to moderately elevated sulphate concentrations. Our study found extremely high levels of sulphate in one site, Trench Pool, which has been shown to suffer from significant urban pollution. Another site, Pinfold Dam, also showed very high concentrations of sulphate (maximum of $\left.113.9 \mathrm{mg} \cdot \mathrm{L}^{-1}\right)$.

\section{$>$ OXYGEN (DO)}

Some populations of $A$. pallipes are found in waters of high oxygen levels (Figure 1i). However, several studies (e.g. Trouilhé et al., 2007) also found populations to persist at concentrations lower than the means cited for European rivers. It appears that there may be a lower threshold for dissolved oxygen concentration below which crayfish are not found (i.e. c. $3-3.5 \mathrm{mg} \cdot \mathrm{L}^{-1}$ ). Some sites within our study might suggest that $A$. pallipes is associated with elevated dissolved oxygen, but we also recorded low DO in some sites (e.g. $4.5 \mathrm{mg} \cdot \mathrm{L}^{-1}$ in the River Derwent, which was flooded at the time, suggesting that low DO may have been temporary).

\section{$>$ NITRATE $\left[\mathrm{NO}_{3}^{-}\right]$}

The majority of $A$. pallipes populations have been found in waters below or close to the average UK, European and global river means for nitrate (Figure 1j). Three notable exceptions relative to European rivers have been documented, however. Broquet et al. (2002), Trouilhé et al. (2003) and Trouilhé et al. (2007) have reported some French populations of $A$. pallipes to inhabit waters with substantially higher nitrate concentrations than other authors. Our field survey found a range of nitrate concentrations in waters inhabited by $A$. pallipes very similar to those of European rivers. Five sites had nitrate concentrations greater than $10 \mathrm{mg} \cdot \mathrm{L}^{-1}$, with three of these exceeding $15 \mathrm{mg} \cdot \mathrm{L}^{-1}$.

\section{$>$ PHOSPHATE $\left[\mathrm{PO}_{4}^{3-}\right]$}

Figure $1 \mathrm{k}$ displays phosphate concentrations of waters inhabited by $A$. pallipes. Most populations have been found at low phosphate concentrations relative to UK and European rivers, but one study has found the species in concentrations at the higher end of UK and European river means in Italy (Favaro et al. , 2010). Our study found English populations in water with low phosphate concentration. Populations were found in a narrow range that lies below those found in some published studies. 
$>P H$

A. pallipes populations are found in the literature across a range of $\mathrm{pH}$ values (e.g. $\mathrm{pH} 6.0$ to 9.2 in Britain; Jay and Holdich, 1981) (Figure 1I). These values lie around the range of cited means for UK and European rivers, but lie in the higher range of means for global rivers. Populations were not found to occur in water of $\mathrm{pH}$ below 6.0.

\section{> TOTAL SUSPENDED SOLIDS (TSS) AND SILTATION}

Only four studies have investigated total suspended solids (TSS) levels in waters inhabited by $A$. pallipes (Figure $1 \mathrm{~m}$ ). Whilst three of these reported populations in levels lower than the average of European and global river means (Foster, 1995; Broquet et al., 2002; Trouilhé et al., 2003), one study found populations persisting in water with concentrations of TSS up to $489.3 \mathrm{mg} \cdot \mathrm{L}^{-1}$ (Trouilhé et al., 2007), far higher than the means found in a review of European and global river chemistry. This maximum refers to one site inhabited by crayfish. No information exists regarding the long term status of this population, and this level of TSS may be a result of habitat degradation. A laboratory study by Rosewarne et al. (2014) found that levels above $500 \mathrm{mg} \cdot \mathrm{L}^{-1}$ resulted in gill fouling in all exposed crayfish (A. pallipes), whilst $250 \mathrm{mg} \cdot \mathrm{L}^{-1}$ was associated with fouling in $92 \%$ of exposed individuals. However, the same study did not find any reduction in survival over 45 days in concentrations up to $1000 \mathrm{mg} \cdot \mathrm{L}^{-1}$, indicative of at least short-term tolerance for extremely high levels. The other six sites in the study lie well within the range of European river means. It therefore seems apparent that crayfish can persist in waters with a range of TSS. No conclusions, however, can be made about the adverse effects of siltation on $A$. pallipes distribution. Elevated TSS levels in waters inhabited by crayfish populations in France (Trouilhé et al. , 2007) are associated with elevated nitrate and potassium concentrations, and a lower range of $\mathrm{pH}$ values. This is indicative of more polluted waters, and lends support for some populations of $A$. pallipes persisting in lower water quality for certain variables.

\section{DISCUSSION}

A summary of the conclusions from the review and water chemistry analysis is shown in Table III. A. pallipes appears to be fairly tolerant to a range of conditions for of a number of water chemistry variables. Rallo and Garcia-Arberas (2002) carried out multivariate analyses of a variety of variables for waters inhabited and uninhabited by crayfish in Spain. From their analysis sulphate and magnesium ions were the only factors that discriminated between crayfish presence/absence. In contrast, the current study indicates that the magnesium concentration of waters inhabited by crayfish is similar to the range of European means, and is unlikely to influence the distribution of crayfish populations. Similarly, $A$. pallipes does appear to associate with lower sulphate values than those reported for major European rivers. From our review of the literature and our field study, some factors that may be associated with crayfish presence relative to European and global means are: low conductivity; low ammonium; low sodium; low sulphate; low nitrate; low phosphate; and high dissolved oxygen. These variables can be grouped into those relating to anthropogenic inputs and those important for ecdysis and the production of the crustacean exoskeleton.

\section{> HUMAN-INFLUENCED WATER CHEMISTRY}

Conductivity is a correlate for nutrient load, and can indicate geology, watershed size, and the presence of mine waste or waste water (Goldenberg et al., 1984; García-Criado et al., 1999; Gucht et al., 2005). A. pallipes populations appear to be associated with low values of conductivity relative to European and global means. It is likely that the observed association 


\section{Table III}

Summary of conclusions from review of literature regarding waters inhabited by $A$. pallipes and analysis of water samples from UK populations of the species.

\begin{tabular}{|l|l|}
\hline Variable & \multicolumn{1}{|c|}{ Conclusion } \\
\hline Conductivity & Association with low levels $\left(<500 \mu \mathrm{S} \cdot \mathrm{cm}^{-1}\right)$. Upper threshold of $945 \mu \mathrm{S} \cdot \mathrm{cm}^{-1}$. \\
\hline Magnesium & No clear association. Tolerance of high concentration $\left(28.8 \mathrm{mg} \cdot \mathrm{L}^{-1}\right)$. \\
\hline Calcium & No clear association. Populations present at low levels $\left(1.0 \mathrm{mg} \cdot \mathrm{L}^{-1}\right)$. \\
\hline Potassium & No clear association. Tolerance of high concentration $\left(12.2 \mathrm{mg} \cdot \mathrm{L}^{-1}\right)$. \\
\hline Ammonium & Association with low levels $\left(<0.15 \mathrm{mg} \cdot \mathrm{L}^{-1}\right)$. Upper threshold of $0.74 \mathrm{mg} \cdot \mathrm{L}^{-1}$. \\
\hline Sodium & $\begin{array}{l}\text { Association with low levels }\left(<18 \mathrm{mg} \cdot \mathrm{L}^{-1}\right) . \text { Tolerance of high concentration } \\
\left(50.3 \mathrm{mg} \cdot \mathrm{L}^{-1}\right) .\end{array}$ \\
\hline Chloride & No clear association. Upper threshold of $85.2 \mathrm{mg} \cdot \mathrm{L}^{-1}$. \\
\hline Sulphate & $\begin{array}{l}\text { Possible association with low levels }\left(<25 \mathrm{mg} \cdot \mathrm{L}^{-1}\right) \cdot \text { Tolerance of high } \\
\text { concentration }\left(266.8 \mathrm{mg} \cdot \mathrm{L}^{-1}\right) .\end{array}$ \\
\hline Dissolved Oxygen & Association with high levels. Lower threshold of 3.4 mg. $\mathrm{L}^{-1} \mathrm{plausible.}$ \\
\hline Nitrate & $\begin{array}{l}\text { Possible association with low levels }\left(<9 \mathrm{mg} \cdot \mathrm{L}^{-1}\right) . \text { Tolerance of high } \\
\text { concentration }\left(57.2 \mathrm{mg} \cdot \mathrm{L}^{-1}\right) .\end{array}$ \\
\hline Phosphate & $\begin{array}{l}\text { Association with low levels }\left(<0.22 \mathrm{mg} \cdot \mathrm{L}^{-1}\right) \cdot \mathrm{Tolerance} \text { of moderate } \\
\text { concentration }\left(0.39 \mathrm{mg} \cdot \mathrm{L}^{-1}\right) .\end{array}$ \\
\hline pH & No clear association. Only found between pH 6.0 and 9.2. \\
\hline TSS & $\begin{array}{l}\text { Possible association with low levels }\left(<34 \mathrm{mg} \cdot \mathrm{L}^{-1}\right) . \text { Tolerance of high } \\
\text { concentration }\left(489 \mathrm{mg} \cdot \mathrm{L}^{-1}\right) .\end{array}$ \\
\hline
\end{tabular}

patterns reflect pollution, and that crayfish presence is also associated with low levels of other pollution-indicators.

Ammonium, a waste product of animal metabolism, is indicative of agricultural pollution resulting from fertiliser runoff and sewage and is toxic in high concentrations (Berner and Berner, 1996). A. pallipes is associated in general with low ammonium concentrations: 8 of 10 studies found populations restricted to concentrations below $0.15 \mathrm{mg} \cdot \mathrm{L}^{-1}$.

The primary source of nitrate in freshwater ecosystems is surface runoff; from the application of fertilisers in agriculture, and runoff from waste disposal sites and industrial practices (Camargo et al., 2005). Between one third (Meybeck, 1982) and two thirds (Wollast, 1993) of all riverine total dissolved nitrogen $\left(\mathrm{NO}_{3}^{-}\right.$and $\left.\mathrm{NH}^{4+}\right)$ results from pollution. It is also generated in situ via the nitrification of ammonia in sewage (Abeliovich, 1985). Toxicity of nitrate ions has been shown to occur via the conversion of oxygen carrying pigments (haemoglobin and haemocyanin) to forms that are unable to carry oxygen (methaemoglobin and methaemocyanin) (Camargo et al. , 2005). Nitrate is less toxic to aquatic organisms than ammonia or nitrite (Romano and Zeng, 2007). Nevertheless, nitrate concentrations of $10 \mathrm{mg} \cdot \mathrm{L}^{-1}$ are detrimental to some freshwater invertebrates, fish, and amphibians (reviewed by Camargo et al., 2005). Laboratory studies of tolerance demonstrate the ability of crayfish to withstand shortterm exposure to nitrate levels as high as $1000 \mathrm{mg} \cdot \mathrm{L}^{-1}$ (Meade and Watts, 1995), but these findings to not directly relate to long term tolerance in the wild. Whilst some studies have found $A$. pallipes to be associated with low concentrations of nitrate in the wild, three published studies along with the results herein show that populations can persist in very high nitrate levels.

Phosphorus, in the form of the inorganic phosphate ion, plays a vital role in the structure of DNA/RNA, the structure of cells (as phospholipids), and in energy transfer (as adenosine triphosphate or ATP), and is often a limiting nutrient in rivers and lakes (Elser et al., 2007). On the other hand, elevated phosphorous as a result of fertilisers, industrial pollution, and deforestation may lead to eutrophication, particularly in lentic waters (Schindler, 1971). A. pallipes populations are generally found in waters of low phosphate concentration relative to European river means and our field survey data concur with the majority of the literature to suggest that white-clawed crayfish are associated with low phosphate concentrations. 
Chloride is linked with ammonium and sodium; elevated levels of all three variables are associated with polluted waters. Sodium is a vital component of all animal cells, being the primary cation of extracellular fluids. High sodium concentration, however, can cause elevated mortality and limit growth (Hamilton et al., 1975; Heath, 1977). Sodium enters rivers via the weathering of halite $(\mathrm{NaCl})$ and plagioclase $\left(\mathrm{NaAlSi}_{3} \mathrm{O}_{8}\right)$ rocks, from cyclic (sea) salt, and a substantial amount from pollution, such as domestic and industrial sewage and road salt (Meybeck, 1979). Elevated sodium concentrations are associated with elevated chloride and ammonium levels, and are typical of polluted or saline waters. Chloride originates from a range of sources; (i) sea salt; (ii) halite $(\mathrm{NaCl})$ weathering and subsequent dissolution; (iii) volcanic springs; (iv) saline crust dissolution in deserts; (v) pollution. It has been estimated that around $30 \%$ of chloride in the world's rivers is the result of pollution (Meybeck, 1979). Populations of $A$. pallipes appear to be correlated with low concentrations of both sodium and chloride; these observations are in accord with work suggesting that the species may be sensitive to pollution from sewage or industrial effluent (e.g. Reynolds et al., 2002).

Sulphate is a common ion in freshwater environments, but is generally found at low concentrations. There are two major sources of sulphate in rivers; weathering of rocks produces approximately 33 percent (Berner and Berner, 1996) and pollution produces approximately 54 percent (Meybeck, 1979) of global sulphate. Sources of sulphate pollution include acid rain, dry fallout, and fertilisers, particularly in European rivers (Oden and Ahl, 1978). The majority of A. pallipes populations in Europe are found in waters with relatively low sulphate concentration relative to European and global means. Rallo and Garcia-Arberas (2002), however, found populations associated with a wider range of sulphate concentrations, similar to those of European river means. Similarly, our field study found populations of $A$. pallipes in urban areas in relatively high concentrations of sodium, suggesting that the species is not particularly sensitive to sodium pollution alone. However, the species is associated with lower sulphate levels, and hence lower pollution, but does not indicate intolerance for levels typically observed in rivers throughout Europe.

A number of crayfish species (for example, Parastacus defossus and Procambarus clarkii) have been observed living in the very low or anoxic conditions associated with muddy habitats and are, to an extent, physiologically adapted to low dissolved oxygen (DO) conditions (reviewed by McMahon, 2002). Relatively few studies have examined dissolved oxygen levels in waters inhabited by $A$. pallipes. Three studies found the species associated with high levels relative to European and global means, whilst Trouilhé et al. (2007) found crayfish across a wide range of DO concentrations. Our field results similarly demonstrate a wide range, but also support an association with elevated DO. The results of our review, however, may challenge the theoretical minimum dissolved oxygen tolerance of around $5 \mathrm{mg} \cdot \mathrm{L}^{-1}$ (Trouilhé et al., 2007). This suggests that whilst an association with raised DO may exist, the species is tolerant, to a certain degree, of lower values.

Elevated hydrogen ion concentration (i.e. low $\mathrm{pH}$ ) is toxic to many freshwater invertebrates (e.g. Bell, 1970). Low pH is believed to result in reduced growth by impairing the conversion efficiency of food energy for use in growth (Lee et al., 1983), and Seiler and Turner (2004) found growth rates of the North American crayfish Cambarus bartonii to be higher in neutral than in acidic waters. In laboratory studies, $C$. bartonii adults were found to have an LD50 at $\mathrm{pH} 2.43$ (Distefano et al., 1991). A. pallipes were shown in laboratory studies to suffer high mortality at $\mathrm{pH}$ less than 6.0 in long-term studies (Jay and Holdich, 1977). The studies reviewed herein indicate that $A$. pallipes is tolerant of $\mathrm{pH}$ from 6 to 9.2, although populations are generally associated with $\mathrm{pH}$ from 7.5 to 8.5.

In nature, higher levels of individual variables may be associated with other variables that together result in toxicity and elevated mortality. For example, crayfish may be tolerant of high concentrations of ammonium alone, but in rivers, elevated ammonium may be associated with raised levels of other pollutants such as heavy metals, nitrate, sulphate, sodium, and chloride, and with low levels of dissolved oxygen. Therefore, whilst some studies have found an association of $A$. pallipes with low values of certain water chemistry variables, it may be a combination of several pollution-indicating variables that limit the distribution of the species. 


\section{> EXOSKELETONS AND ECDYSIS}

Magnesium is an essential element required in crustacean integuments, and is required for successful ecdysis (moulting) (Jussila et al., 1995). The crustacean exoskeleton is composed of chitin encrusted with calcium carbonate, making calcium another important metal ion in crayfish development (Roer and Dillaman, 1984). Potassium is also used in animal physiology, including action potentials of neurons and membrane polarisation (Roer and Dillaman, 1984). Comparing magnesium values for waters inhabited by $A$. pallipes with those for European and global means suggests no discernable pattern. Whilst crustaceans have a requirement for magnesium, it is unlikely to be limiting in these environments. This is particularly indicated by the presence of crayfish in water with very low magnesium concentration.

Various authors have suggested a lower limit of $5 \mathrm{mg} \cdot \mathrm{L}^{-1}$ calcium concentration (Greenaway, 1974), as supported by observations of Jay and Holdich (1981) in the British Isles, and Trouilhé et al. (2007) in France. Furthermore, laboratory manipulations involving Astacus astacus by Rukke (2002) showed stunted growth and reduced survival below $5 \mathrm{mg} \cdot \mathrm{L}^{-1}$ calcium compared to that at $10 \mathrm{mg} \cdot \mathrm{L}^{-1}$. Survival of the Parastacid crayfish Paranephrops zealandicus was increased as the concentration of calcium in laboratory investigations exceeded $10 \mathrm{mg} \cdot \mathrm{L}^{-1}$ (Hammond et al. , 2006). However, survival of crayfish has been observed in waters with calcium concentrations as low as $2 \mathrm{mg} \cdot \mathrm{L}^{-1}$ for Orconectes virilis (France, 1987), and A. astacus (e.g. Jussila et al., 1995). These observations suggest that calcium is not limiting crayfish distribution. Crayfish are found over a wide range of potassium concentrations and their distribution is therefore unlikely to be affected by potassium concentration within the rivers examined. However, extreme levels of potassium caused by pollution may still adversely affect crayfish. Further research is needed to rule this possibility out.

\section{> RECOMMENDATIONS FOR CRAYFISH CONSERVATION}

In general our results suggest that $A$. pallipes populations are restricted to habitats that do not receive significant sewage effluent or contamination: typically, waters low in conductivity, sodium, chloride, nitrate, ammonium, to a lesser extent sulphate, and high dissolved oxygen concentration. The water chemistry data reviewed and analysed in this study form only a snapshot of the chemical conditions. Whilst these data are likely to give an indication of long term conditions, water chemistry will vary over time. Caution must therefore be exercised when making conclusions from single time point measurements of both water chemistry and crayfish presence. Long term studies of chemical conditions and crayfish abundance are necessary to allow for conclusions to be made regarding suitable conditions for the maintenance of wild populations. Such studies have not been well documented in the literature, but they are of great importance. We strongly recommend that these records be established and made available for the wider conservation audience. Water chemistry is not the only factor affecting the distribution of $A$. pallipes populations, which has also become restricted in because of invasive crayfish and crayfish plague. An understanding of the association of white-clawed crayfish with specific water chemistry variables, however, can assist in locating suitable Ark Sites for the relocation of threatened populations.

At present, the conservation of endangered populations of $A$. pallipes in the UK is believed to heavily depend upon the success of relocation to Ark Sites (e.g. Kemp et al., 2000; Whitehouse et al., 2009; Haddaway, 2010). Based on the analysis of the papers reviewed here, we propose guidelines for suitable water chemistry of Ark Sites (Figure 3). Ideally, these recommendations should be supported by manipulation studies to investigate the impact of different water chemistry on crayfish survival, growth, and reproduction. This may not be easy, however, since the toxicity of single water chemistry variables may not relate to habitat associations in practice. For example, Figure 2 demonstrates how concentrations of variables are often linked in rivers, in this case in the River Wharfe. Sources of agricultural (site 3) and industrial/urban runoff (sites 14 and 15) are evidenced in all three variables shown. Furthermore, the need to identify suitable Ark Sites is pressing due to the rapid migration of invasive 


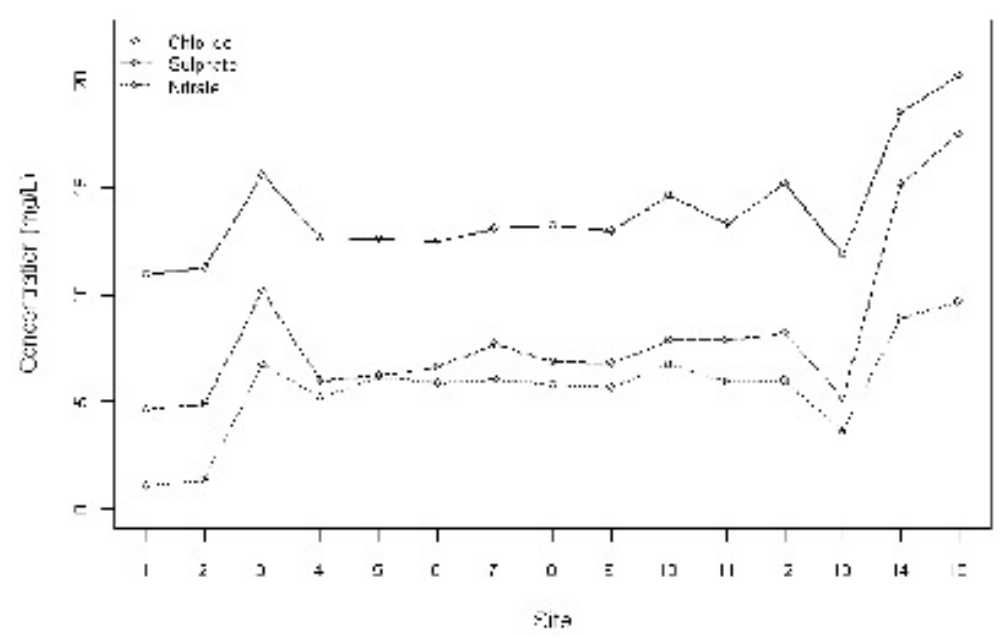

\section{Figure 2}

Concentration of chloride, sulphate, and nitrate in the River Wharfe from upstream (site 1) to 676 downstream (site 15) (E. Imhoff, R. Mortimer, and A. Dunn unpublished data).

crayfish and Chinese mitten crab, which has also recently been suggested as a possible vector for crayfish plague (Aphanomyces astaci) (Svoboda et al., 2014), and on-going habitat degradation. In practice, it is often necessary to relocate threatened populations over short timescales.

In order to make recommendations to identify Ark Sites where water chemistry is suitable, data in this review and the results of field measurements were used to plot ranges of variables where crayfish occur and provide baselines that may form guidelines for water quality. Figure 3 displays these guidelines for water quality of Ark Sites by presenting variable ranges, grand means (means of study means), and the minima and maxima for study means (minimum mean and maximum mean). In order to be certain that water chemistry of an Ark Site is suitable, water chemistry should be measured and variables should fall within the narrower buffer zone, corresponding to the range of study means for variables measured within the literature. We believe that this represents a reliable buffer zone for acceptable water quality, since populations of $A$. pallipes have been recorded within these values.

\section{CONCLUSIONS}

This review and empirical study highlights several variables that may have thresholds dictating crayfish distribution. These variables are; conductivity, ammonium, sodium, dissolved oxygen and phosphate, and to a lesser extent sulphate, nitrate, and total suspended solids. There is substantial variability in many variables between studies and between sites in the same study. The presence of populations in the extremes of some variables may demonstrate a tolerance for certain types of pollution, for example urban pollution, but further investigation of these populations is paramount. Investigations of the water chemistry associations of $A$. pallipes populations have successfully characterised many waters containing the species. However, such studies are now highly unlikely to find water chemistry as a causative factor discriminating the presence and absence of crayfish. Population losses due to crayfish plague and competition with invasive non-native crayfish will overshadow all but drastic pollution events. Whilst some populations of $A$. pallipes may persist over a wide range of certain variables, recommendations can be made for habitat restoration and relocation programs allowing the identification of habitats that are likely to be within acceptable ranges for threatened populations of white-clawed crayfish. The recommendations included herein are intended as a starting point for the selection of Ark Sites for relocation conservation. 
N.R. Haddaway et al.: Knowl. Managt. Aquatic Ecosyst. (2015) 416, 01
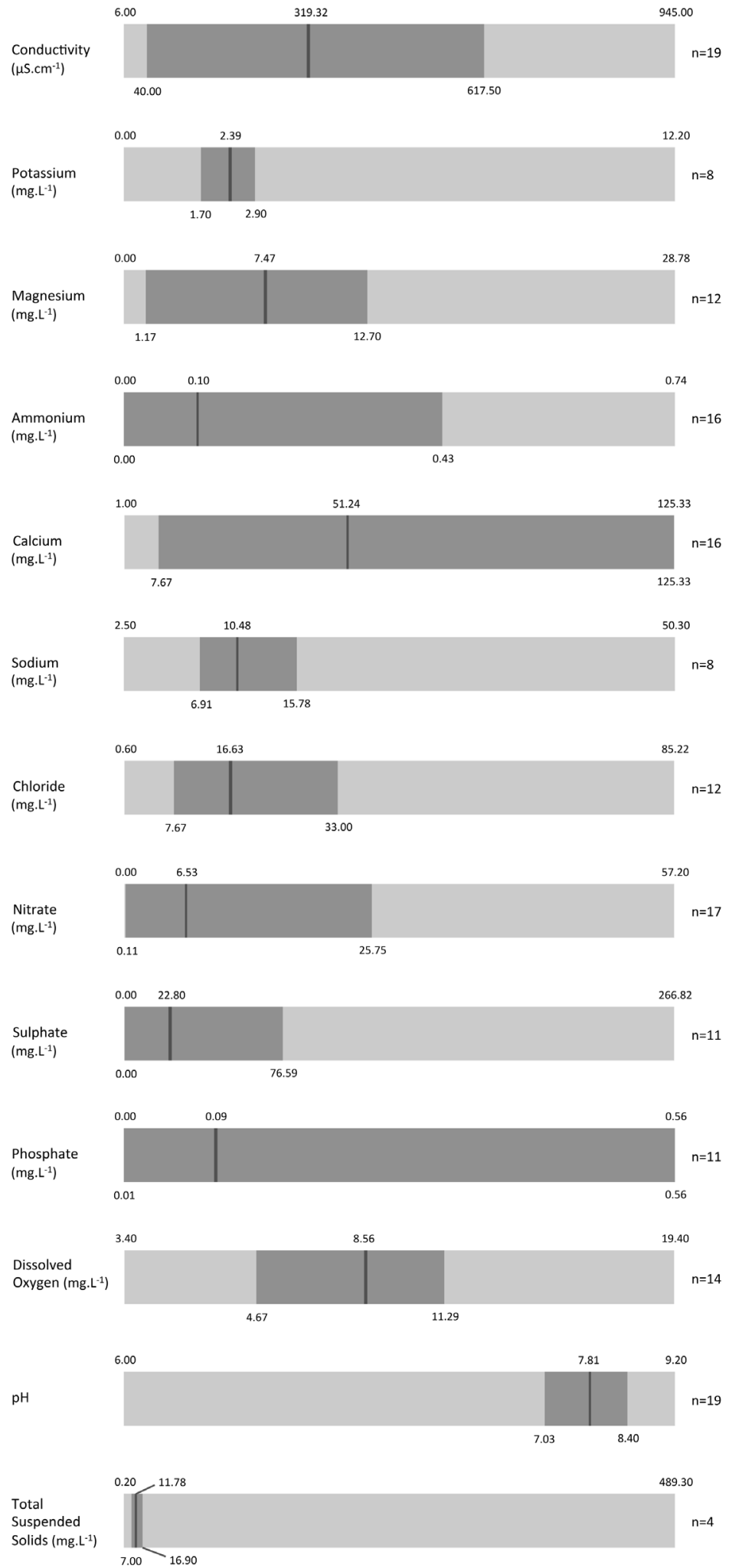

\section{Figure 3}

Recommended water quality guidelines for $A$. pallipes within the literature and from the study herein. The extremities (upper left and upper right numbers) represent the absolute ranges, the black bar (upper middle number) represent the mean mean values, and the intermediate values (lower left and lower right numbers) represent the mean minimum and mean maximum values respectively. All values are in $\mathrm{mg} \cdot \mathrm{L}^{-1}$ with the exception of conductivity $\left(\mu \mathrm{S} \cdot \mathrm{cm}^{-1}\right)$ and $\mathrm{pH}$. 


\section{ACKNOWLEDGEMENTS}

This research was funded by the National Environmental Research Council (NERC) and the Environment Agency. Thanks to Stephanie Peay for informative discussions about Ark Sites.

\section{REFERENCES}

Abeliovich A., 1985. Nitrification of ammonia in wastewater: Field observations and laboratory studies. Water Res., 19, 1097-1099.

Arrignon J.C.V. and Roché B., 1983. Population of the crayfish Austropotamobius pallipes pallipes Lereb. in a brook of Corsica, France. Freshwater Crayfish 5, 229-238.

Arrignon J.C.V., Lamy G. and Roché B., 1993. Situation d'une population corse d' Austropotamobius pallipes pallipes Lereb. Publ. Assoc. Dev. Aquac. 35, 89-98.

Baldy, V., Gobert, V., Guerold, F., Chauvet, E., Lambrigot, D. and Charcosset, J.Y., 2007. Leaf litter breakdown budgets in streams of various trophic status: effects of dissolved inorganic nutrients on microorganisms and invertebrates. Freshw. Biol., 52, 1322-1335.

Barbaresi S., Cannicci S., Vannini M. and Fratini S., 2007. Environmental correlates of two macrodecapods distribution in Central Italy: Multi-dimensional ecological knowledge as a tool for conservation of endangered species. Biol. Cons., 136, 431-441.

Bell H.L. 1970. Effects of pH on the life cycle of the midge Tanytarsus dissimilis. Can. Entomol., 102, 636-639.

Berner E.K. and Berner R.A., 1996. Global Environment: Water, Air, and Geochemical Cycles. PrenticeHall Inc., Upper Saddle River, New Jersey.

Broquet T., Thibault M. and Neveu A., 2002. Distribution and habitat requirements of the white clawedcrayfish, Austropotamobius pallipes, in a stream from the pays de Loire region, France: an experimental and descriptive study. Bull. Fr. Pêche Piscic., 367, 717-728.

Brusconi S., Bertocchi S., Renai B., Scalici M., Souty-Grosset C. and Gherardi, F., 2008. Conserving indigenous crayfish: stock assessment and habitat requirements in the threatened Austropotamobius italicus. Aquat. Conserv., 18, 1227-1239.

Camargo J.A., Alonso A. and Salamanca A., 2005. Nitrate toxicity to aquatic animals: a review with new data for freshwater invertebrates. Chemosphere 58, 1255-1267.

Demers A. and Reynolds, J.D., 2002. A survey of the white-clawed crayfish, Austropotamobius pallipes (Lereboullet), and of water quality in two catchments of eastern Ireland. Bull. Fr. Pêche Piscic., $367,729-740$.

Distefano R.J., Neves R.J., Helfrich L.A. and Lewis M.C., 1991. Response of the crayfish Cambarus bartonii bartonii to acid exposure in southern Appalachian streams. Can. J. Zool., 69, 1585-1591.

Elser J.J., Bracken M.E.S., Cleland E.E., Gruner D.S., Harpole W.S., Hillebrand H., Ngai J.T., Seabloom E.W., Shurin J.B. and Smith J.E., 2007. Global analysis of nitrogen and phosphorus limitation of primary producers in freshwater, marine and terrestrial ecosystems. Ecol. Lett., 10, 1135-1142.

Favaro L., Tirelli T. and Pessani D., 2010. The role of water chemistry in the distribution of Austropotamobius pallipes (Crustacea Decapoda Astacidae) in Piedmont (Italy). C.R. Biol., 333, 68-75.

Favaro L., Tirelli T. and Pessani D. 2011. Modelling habitat requirements of white-clawed crayfish (Austropotamobius pallipes) using support vector machines. Knowl. Managt. Aquatic Ecosyst., 401, 21.

Foster J., 1995. Factors influencing the distribution and abundance of the crayfish Austropotamobius pallipes (Lereboullet) in Wales and the Marches, UK. Freshwater Crayfish, 8, 78-98.

France R.L. 1987. Calcium and trace metal composition of crayfish (Orconectes virilis) in relation to experimental lake acidification. Can. J. Fish. Aquat. Sci., 44, 107-113.

Füreder L. and Reynolds J.D., 2003. Is Austropotamobius pallipes a good bioindicator? Bull. Fr. Pêche Piscic., 370-371, 157-163.

Füreder L., Oberkofler B., Hanel R., Leiter J. and Thaler B., 2003. The freshwater crayfish Austropotamobius pallipes in South Tyrol: Heritage species and bioindicator. Bull. Fr. Pêche Piscic., 370-371, 79-95. 
Garcia-Arberas L. and Rallo A., 2000. Survival of natural populations of Austropotamobius pallipes in rivers in Bizkaia, Basque Country (North of Iberian Peninsula). Bull. Fr. Pêche Piscic., 356, 17-30.

García-Criado F., Tomé A., Vega F.J. and Antolin C., 1999. Performance of some diversity and biotic indices in rivers affected by coal mining in northwestern Spain. Hydrobiologia, 394, 209-217.

Gherardi F. and Holdich D.M., 1999. Crayfish in Europe as alien species: how to make the best of a bad situation? A.A. Balkema, Rotterdam/Brookfield.

Goldenberg L.C., Magaritz M., Amiel A.J. and Mandel S., 1984. Changes in hydraulic conductivity of laboratory sand-clay mixtures caused by a seawater-freshwater interface. J. Hydrol., 70, 329-336.

Gottstein S., Kerovec M., Maguire I. and Bukvić I., 1999. Ecological notes on Austropotamobius pallipes italicus (Faxon, 1914)(Decapoda, Astacidae) in a karstic spring of the Neretva Delta (Croatia). Freshwater Crayfish, 12, 620-628.

Grandjean F., Frelon-Raimond M., and Souty-Grosset C., 2002. Compilation of molecular data for the phylogeny of the genus Austropotamobius: one species or several? Bull. Fr. Pêche Piscic., 367, 671-680.

Grandjean, F., Jandry, J., Bardon, E., Coignet, A., Trouilhé, M.C, Parinet, B., Souty-Grosset, C. and Brulin, M., 2011. Use of Ephemeroptera as bioindicators of the occurrence of the white-clawed crayfish (Austropotamobius pallipes). Hydrobiologia, 671, 253-258.

Greenaway P., 1974. Calcium balance at the post-molt stage of the freshwater crayfish Austropotamobius pallipes. J. Exp. Biol., 61, 35-45.

Gucht K., Vandekerckhove T., Vloemans N., Cousin S., Muylaert K., Sabbe K., Gillis M., Declerk S., Meester L. and Vyverman W., 2005. Characterization of bacterial communities in four freshwater lakes differing in nutrient load and food web structure. FEMS Microbiol. Ecol., 53, 205-220.

Haddaway, N.R., 2010. Conservation of British white-clawed crayfish (Austropotamobius pallipes) using Ark Sites Ph.D. Thesis, University of Leeds.

Hamilton R.W., Buttner J.K. and Brunetti R.G., 1975. Lethal levels of sodium chloride and potassium chloride for an oligochaete, a chironomid midge, and a caddisfly of Lake Michigan. Environ. Entomol., 4, 1003-1006.

Hammond K.S., Hollows J.W., Townsend C.R. and Lokman P.M., 2006. Effects of temperature and water calcium concentration on growth, survival and moulting of freshwater crayfish, Paranephrops zealandicus. Aquaculture, 251, 271-279.

Heath A.G., 1977. Toxicity of intermittent chlorination to freshwater fish: influence of temperature and chlorine form. Hydrobiologia, 56, 39-47.

Holdich D.M., 1996. Austropotamobius pallipes In: Van Helddingen P.J., Willemse I. and Speight M.C.D., (eds.). Background information on invertebrates of the Habitats Directive and the Bern Convention. Part I - Crustacea, Coleoptera and Lepidoptera. Nature and environment 79. Council of Europe Publishing, Strasbourg.

Holdich D.M. and Reeve I.D., 1991. The distribution of freshwater crayfish in the British Isles with particular reference to crayfish plague, alien introductions and water quality. Aquat. Conserv., 1, 139-158.

Holdich D.M., Rogers W.D. and Reynolds J.D., 1999. Native and alien crayfish in the British Isles. Pp. 221-270 In: Gherardi F. and Holdich D.M. (eds.). Crayfish in Europe as Alien Species: How to Make the Best of a Bad Situation? Balkema, Rotterdam/Brookfield.

Holdich D.M., Palmer M. and Sibley P.J., 2009. The indigenous status of Austropotamobius pallipes (Lereboullet) in Britain. Pp. 1-11 In: Brickland J., Holdich D.M. and Imhoff E.M. (eds.). Crayfish Conservation in the British Isles, Proceedings of conference held in Leeds.

IUCN, 2011. IUCN Red List of Threatened Species. Version 2011.1. Downloaded on 27 July 2011.

Jalali S. and Wohlin C., 2012. Systematic literature studies: database searches vs. backward snowballing. Proceedings of the ACM-IEEE international symposium on Empirical software engineering and measurement, 29-38.

Jandry, J., Parinet, B., Brulin, M., and Grandjean, F., 2014. Ephemeroptera communities as bioindicator of white-clawed crayfish: in restocking attempts. Ecol. Indic., 46, 560-565.

Jay D. and Holdich D.M., 1977. The $\mathrm{pH}$ tolerance of the crayfish Austropotamobius pallipes (Lereboullet). Freshwater Crayfish, 3, 363-370.

Jay D. and Holdich D.M., 1981. The distribution of the crayfish Austropotamobius pallipes in British waters. Freshw. Biol., 11, 121-130. 
Jussila J., Henttonen P. and Huner J.V., 1995. Calcium, magnesium and manganese content of the noble crayfish Astacus astacus (L.) branchial carapace and its relationships to water and sediment mineral contents of two ponds and one lake in central Finland. Freshwater Crayfish, 10, 230-238.

Kemp E., Birkinshaw N., Peay S. and Hiley P.D., 2000. Reintroducing the White-clawed Crayfish. Conserving Natura 2000 Rivers Conservation Techniques Series No. 1. English Nature, Peterborough.

Kouba A., Petrusek A. and Kozák P. 2014. Continental-wide distribution of crayfish species in Europe: update and maps. Knowl. Managt. Aquatic Ecosyst., 413, 05.

Lee R.M., Gerking S.D. and Jezierska B., 1983. Electrolyte balance and energy mobilization in acid stressed rainbow trout Salmo gairdneri and their relation to reproductive success. Envir. Biol. Fish., 8, 115-124.

Lilley A.J., Brooker M.P. and Edwards R.W., 1979. The distribution of the crayfish, Austropotamobius pallipes (Lerebollet), in the Upper Wye catchment. Nature Wales, 16, 195-200.

Lyons R., and Kelly-Quinn M., 2003. An investigation into the disappearance of Austropotamobius pallipes (Lereboullet) populations in the headwaters of the Nore River, Ireland and the correlation to water quality. Bull. Fr. Pêche Piscic., 370-371, 139-150.

McMahon B.R., 2002. Physiological adaptation to environment. In: Holdich D.M., (ed.) Biology of Freshwater Crayfish. Blackwell Science, Oxford, pp. 327-376.

Meade M.E. and Watts S.A., 1995. Toxicity of ammonia, nitrite, and nitrate to juvenile Australian crayfish, Cherax quadricarinatus. J. Shellfish Res., 14, 341-346.

Meybeck M., 1979. Concentrations des eaux fluviales en elements majeurs et apports en solution aux oceans. Rev. Geol. Dyn. Geogr., 21, 215-246.

Meybeck M., 1982. Carbon, nitrogen and phosphorus transport by world rivers. Am. J. Sci., 282, 401-450.

Nardi P.A., Bernini F., Bo T., Bonardi A., Fea G., Ghia D., Negri A., Razzetti E., Rossi S. and Spairani, M., 2005. Status of Austropotamobius pallipes complex in the watercourses of the Alessandria province (NW Italy). Bull. Fr. Pêche Piscic., 376-377, 585-598.

Oden S. and Ahl T., 1978. The sulphur budget of Sweden. In: Hutchinson T.C. and Havas M. (eds.). Effects of Acid Precipitation on Terrestrial Ecosystems. Plenum Press, New York, pp. 111-122.

Peay S., 2003. Guidance on habitat for white-clawed crayfish and how to restore it. R\&D Technical Report W1-067/TR. Environment Agency, Bristol, 66.

Peay S., 2009. Selection criteria for "Ark Sites" for white-clawed crayfish In: Brickland J.H., Holdich D.M. and Imhoff E.M. (eds.). Crayfish Conservation in the British Isles. Peak Ecology Ltd., Leeds, UK.

Rallo A. and Garcia-Arberas L., 2002. Differences in abiotic water conditions between fluvial reaches and crayfish fauna in some northern rivers of the Iberian Peninsula. Aquat. Living Resour., 15, $119-128$

Renai B., Bertocchi S., Brusconi S., Gherardi F., Grandjean F., Lebboroni M., Parinet B., Grosset C.S. and Trouilhe M.C., 2006. Ecological characterisation of streams in Tuscany (Italy) for the management of the threatened crayfish Austropotamobius pallipes complex. Bull. Fr. Pêche Piscic., 380-381, 1095-1113.

Reyjol Y. and Roqueplo C., 2002. Répartition des écrevisses à pattes blanches, Austropotamobius pallipes (Lereboullet, 1858), dans trois ruisseaux de Corrèze. Bull. Fr. Pêche Piscic., 367, 741-759.

Reynolds J., Gouin N., Pain S., Grandjean F., Demers A. and Souty-Grosset C., 2002. Irish crayfish populations: ecological survey and preliminary genetic findings. Freshwater Crayfish, 13, 551-561.

Roer, R. and Dillaman, R., 1984. The structure and calcification of the crustacean cuticle. Am. Zool., 24, 893-909.

Romano N. and Zeng C., 2007. Acute toxicity of sodium nitrate, potassium nitrate and potassium chloride and their effects on the haemolymph composition and gill structure of early juvenile blue swimmer crabs (Portunas pelagicus, Linneaus 1758) (Decapoda, Brachyura, Portunidae). Environ. Toxico. Chem., 26, 1955-1962.

Rosewarne P.J., Svendsen J.C., Mortimer, R.J.G. and Dunn A.M., 2014. Muddied waters: suspended sediment impacts on gill structure and aerobic scope in an endangered native and an invasive freshwater crayfish. Hydrobiologia, 722, 61-74.

Rukke N.A., 2002. Effects of low calcium concentrations on two common freshwater crustaceans, Gammarus lacustris and Astacus astacus. Funct. Ecol., 16, 357-366. 
Scalici M. and Gibertini G., 2005. Can Austropotamobius italicus meridionalis be used as a monitoring instrument in Central Italy? Preliminary observations. Bull. Fr. Pêche Piscic., 376-377, 613-625.

Schindler D.W., 1971. Carbon, nitrogen, and phosphorus and the eutrophication of freshwater lakes. J. Phycol., 7, 321-329.

Seiler S.M. and Turner A.M., 2004. Growth and population size of crayfish in headwater streams: individual- and higher-level consequences of acidification. Freshw. Biol., 49, 870-881.

Smith G.R.T., Learner M.A., Slater F.M. and Foster J., 1996. Habitat features important for the conservation of the native crayfish Austropotamobius pallipes in Britain. Biol. Cons., 75, 239-246.

Svoboda, J., Strand, D.A., Vrålstad, T., Grandjean, F., Edsman, L., Kozák, P., Kouba, A., Fristad, R.F. Bahadir Koca, S. and Petrusek, A., 2014. The crayfish plague pathogen can infect freshwaterinhabiting crabs. Freshw. Biol., 59, 918-929.

Talley N.L. and Daggett M.A.F., 2006. Determination of small stream water quality using crayfish as a model organism. FASEB Journal, 20, A67.

Troschel H.J., 1997. Distribution and ecology of Austropotamobius pallipes in Germany. Bull. Fr. Pêche Piscic., 347, 639-647.

Trouilhé M.C., Ricard F., Parinet B., Grandjean F. and Souty-Grosset C., 2003. Management of the whiteclawed crayfish (Austropotamobius pallipes) in Western France: Abiotic and biotic factors study. Bull. Fr. Pêche Piscic., 370-371, 97-114.

Trouilhé M.C., Souty-Grosset C., Grandjean F. and Parinet B., 2007. Physical and chemical water requirements of the white-clawed crayfish (Austropotamobius pallipes) in western France. Aquat. Cons., 17, 520-538.

Trouilhé, M-C, Freyssinel, G., Jandry, J., Brulin, M., Parinet, B., Souty-Grosset, C. and Grandjean, F., 2012. The relationship between Ephemeroptera and presence of the white-clawed crayfish (Austropotamobius pallipes). Case study in the Poitou-Charentes (France). Fundam. Appl. Limnol., 179, 293-303.

Trožić-Borovac S., 2011. Freshwater crayfish in Bosnia and Herzegovina: the first report on their distribution. Knowl Managt. Aquatic Ecosyst., 401, 26.

Whitehouse A.T., Peay S. and Kindemba V., 2009. Ark Sites for White-clawed crayfish - guidance for the aggregates industry. Buglife - The Invertebrate Conservation Trust, Peterborough.

Wollast R., 1993. Interactions of carbon and nitrogen cycles in the coastal zone. In: Wollast R., Mackenzie F.T. and Chou L. (eds.). Interactions of C, N, P and S Biogeochemical Cycles and Global Change. Springer-Verlag, Berlin, Heidelberg, 195-210. 


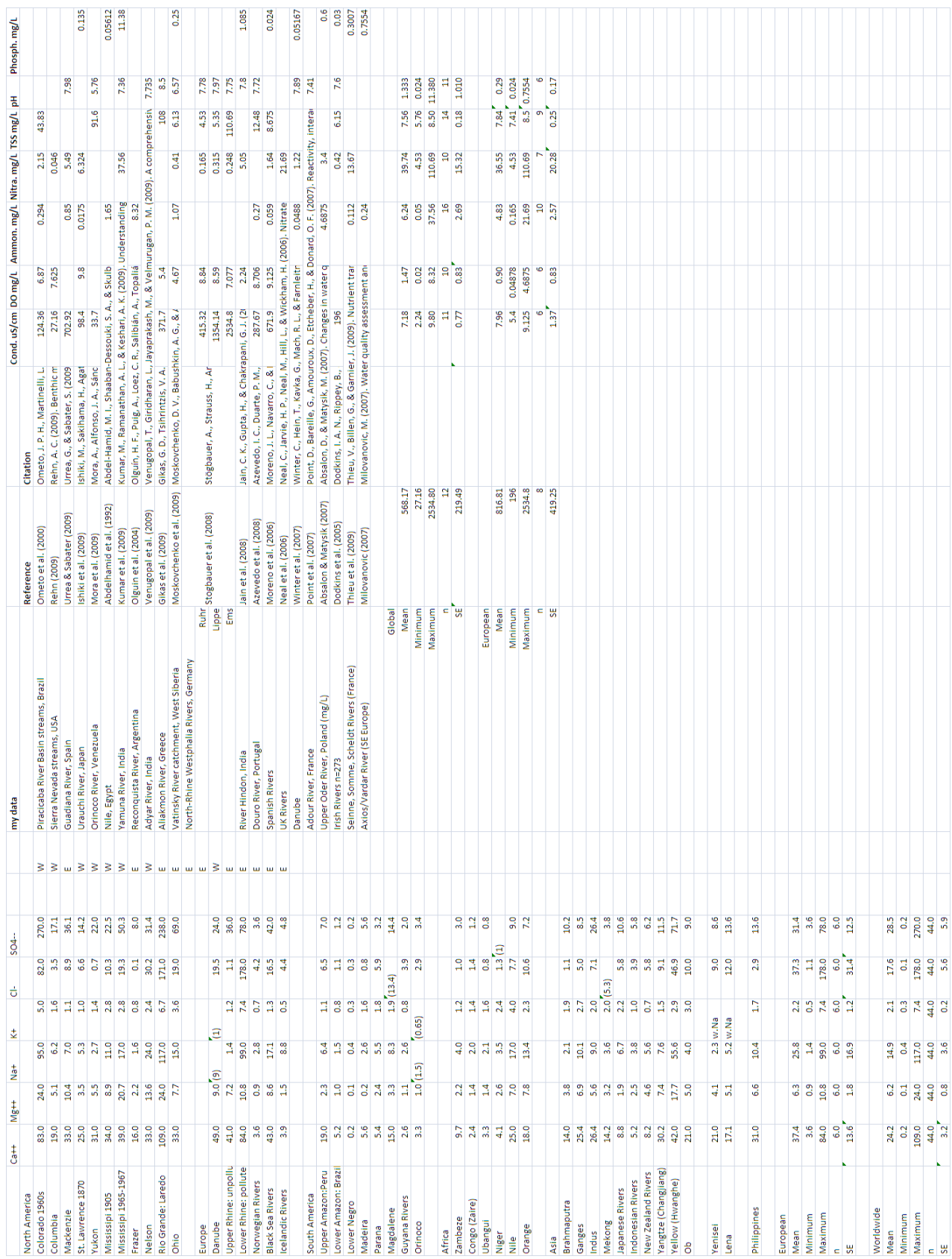


N.R. Haddaway et al.: Knowl. Managt. Aquatic Ecosyst. (2015) 416, 01

\section{Appendix 2.}

Annual average concentrations of selected determinands of river water quality, by river location: 1980, 1990 and $1995-2005$ Great Britain Region Average levels 1980, 1990, and 1995-2005

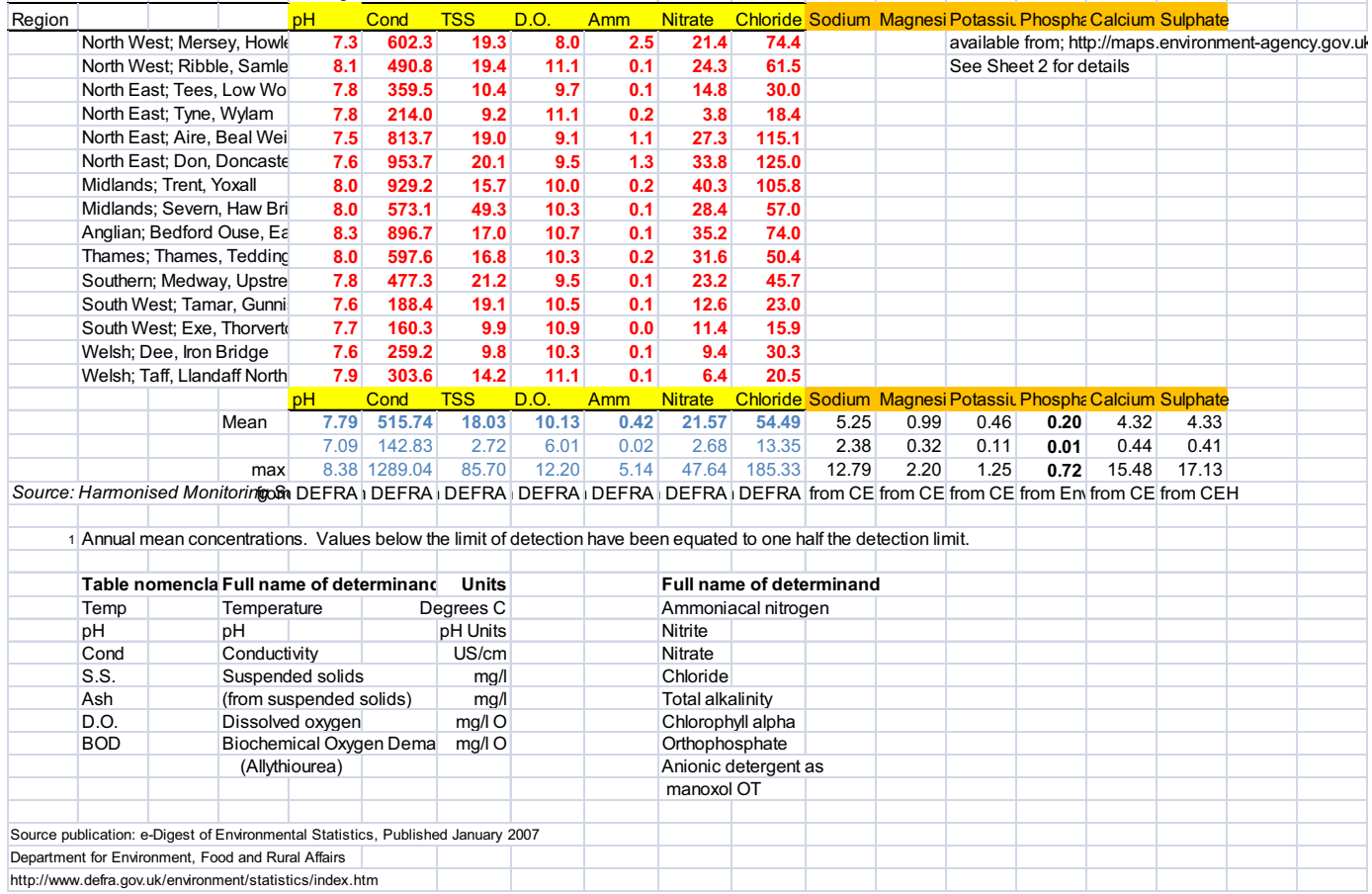


Appendix 2. Continued.

\begin{tabular}{|c|c|c|c|c|c|c|c|c|c|c|c|c|c|c|}
\hline SITENAME & SDATE & $\mathrm{pH}$ & TSS & Conduct ivity & DO & Ammonium & Nitrate & Chloride & Fhos phate & Sulohate & Sodium & Fotgss ium & Calcium & Magnes ium \\
\hline Es thwaite & $2004-01-01$ & 7.58 & & & 10.78 & 0.02 & 0.48 & & 0.01 & & & & & \\
\hline Windermere & $2001.01 \cdot 01$ & 7.43 & & 05.00 & 10.03 & 0.01 & 0.40 & 0.58 & 0.00 & 400 & 4.18 & 0.49 & 5.31 & 0.87 \\
\hline Llyn Llagi & 2003-01-01 & 5.80 & & 26.25 & & & 0.05 & 5.90 & 0.00 & 205 & 3.68 & 0.14 & 0.91 & 0.51 \\
\hline Esthwsite & $2002-01-01$ & 7.52 & & 98.50 & 10.34 & 0.03 & 0.49 & 10.03 & 0.01 & 0.58 & 5.94 & 0.97 & 10.39 & 1.26 \\
\hline W indermere & 2003-01-01 & 7.70 & & & 10.97 & 0.01 & 0.28 & 8.80 & 0.00 & 0.44 & 4.62 & 0.80 & 8.26 & 1.01 \\
\hline Llyn Llagi & 2002.01 .01 & 5.72 & & 27.00 & & & 0.06 & 7.48 & 0.00 & 1.93 & 4.00 & 0.10 & 0.86 & 0.50 \\
\hline W indermare & $1997.01-01$ & 7.45 & & 70.38 & 10.10 & 0.02 & 0.47 & 7.88 & 0.02 & 4.80 & 4.38 & 0.72 & 6.88 & 0.88 \\
\hline W indermere & $1990.01 \cdot 01$ & 7.27 & & 72.25 & 10.59 & 0.05 & 0.61 & 7.67 & 0.03 & 5.32 & 471 & 0.50 & 0.08 & 0.94 \\
\hline Esthwaite & $1998 \cdot 01 \cdot 01$ & 7.30 & & 113.42 & 10.81 & 0.08 & 0.79 & 11.27 & 0.00 & 7.94 & 7.13 & 1.11 & 10.78 & 1.40 \\
\hline Llyn Llagi & $1994.01 \cdot 01$ & 5.23 & & 2390 & & & 0.09 & 483 & 0.01 & 285 & 293 & 038 & $0 \pi$ & 040 \\
\hline Bus n & $1984-01-01$ & 8.08 & 8.01 & 329.24 & 10.27 & 0.08 & 1.79 & 23.73 & 0.09 & 14.24 & & & & \\
\hline Old Lodge & $2002-01-01$ & 5.05 & & 88.50 & & & 0.27 & 17.87 & 0.01 & 8.78 & 9.37 & 0.79 & 270 & 1.48 \\
\hline Bush & 1998-01-01 & 7.91 & 7.16 & 322,43 & 11,18 & 0.11 & 2.83 & 24.51 & 0.09 & 17.10 & & & & \\
\hline Old Lodge & $1998-01 \cdot 01$ & 4.83 & & 90.42 & & & 0.11 & 18.83 & 0.00 & 8.79 & 9.83 & 0.68 & 253 & 1.41 \\
\hline Nant Teyrn & $1958-01-01$ & 4.91 & & 2202 & & 0.02 & 0.14 & 5.38 & 0.00 & 0.78 & 3.17 & 0.19 & 0.83 & 0.41 \\
\hline Trout Beck & $2002 \cdot 01 \cdot 01$ & 8.98 & 2.88 & 72.30 & & 0.04 & 0.08 & 3.48 & 0.00 & 1.35 & 298 & 0.33 & 11.35 & 0.90 \\
\hline Trout Beck & 2003.01 .01 & 8.94 & 1.43 & 94.98 & & 0.05 & 0.11 & 4.12 & 0.00 & 2.17 & 3.03 & 0.40 & 15.48 & 1. 28 \\
\hline Scost Tarn & $2000-01-01$ & 5.08 & & 29.25 & & & 0.20 & 5.55 & 0.00 & 2.38 & 3.20 & 0.23 & 0.47 & 0.48 \\
\hline Llyn Llagi & 2001-01-01 & 6.05 & & 14.50 & & & 0.08 & 3.80 & 0.00 & 1.83 & 200 & 0.13 & 0.75 & 0.40 \\
\hline Windarmere & $1998.01-01$ & 7.20 & & 68.84 & 10.49 & 0.05 & 0.51 & 7.25 & 0.03 & 4.38 & 4.75 & 0.57 & 5.48 & 0.94 \\
\hline Es thwsite & 1998.01 .01 & 7.48 & & 107.58 & 10.25 & 0.03 & 0.68 & 10.30 & 0.01 & 6.33 & 8.25 & 0.99 & 10. 18 & 1.35 \\
\hline Llyn Llagi & 1997.01 .01 & 5.52 & & 25.25 & & & 0.09 & 5.93 & 000 & 2.55 & 3.43 & 0.13 & 0.94 & 0.50 \\
\hline Scost Trn & $1989-01-01$ & 5.01 & & 33.75 & & & 0.28 & 8.58 & 0.00 & 2.75 & 3.78 & 0.38 & 0.86 & 0.56 \\
\hline Scost Trn & 1991-01-01 & 4.85 & & 40.33 & & & 0.41 & 8.10 & 0.00 & 3.23 & 4.53 & 0.34 & 0.85 & 0.88 \\
\hline Scost Tarn & 2001-01-01 & & & & & & & & & & & & & \\
\hline Scost Tan & $2002-01-01$ & 5.08 & & 28.75 & & & 0.24 & 5.30 & 0.00 & 2.38 & 2.85 & 0.24 & 0.47 & 0.47 \\
\hline Es thwaite & $2001 \cdot 01-01$ & 7.60 & & 102.19 & 10.50 & 0.03 & 0.42 & 9.31 & 0.01 & 5.76 & 5.68 & 0.94 & 9.38 & 1.24 \\
\hline W indermere & $2000-01 \cdot 01$ & 7.50 & & 84.85 & 11.01 & 0.14 & 0.32 & 7.30 & 0.03 & 3.92 & 4.31 & 0.45 & 5.48 & 0.91 \\
\hline Scost Tern & $2004 \cdot 01-01$ & 5. 15 & & 28.75 & & & 0.20 & 4.83 & 0.00 & 2.40 & 2.83 & 0.20 & 0.48 & 0.43 \\
\hline Windermere & $1900.01-01$ & 7.30 & & 65.70 & 10.82 & 0.05 & 0.48 & 7.31 & 0.04 & 4.78 & 4.45 & 0.52 & 5.53 & 0.94 \\
\hline Llyn Llagi & 1998-01-01 & 5.53 & & 24.80 & & & 0.28 & 8.78 & 0.00 & 3.28 & 3.83 & 0.13 & 1.11 & 0.88 \\
\hline S cost Trn & $1998.01-01$ & 5.04 & & 32.25 & & & 0.48 & 5.10 & 0.00 & 2.93 & 3.25 & 0.28 & 0.82 & 0.83 \\
\hline Llyn Llagi & $2004.01 \cdot 01$ & 5.86 & & 25.00 & & & 0.09 & 5.48 & 0.01 & 1.78 & 285 & 0.11 & 0.82 & 0.44 \\
\hline Es thwaite & $1996-01-01$ & 7.23 & & 103.18 & 9.91 & 0.05 & 0.88 & 11.32 & 0.00 & 7.10 & 8.02 & 0.94 & 10.12 & 1.29 \\
\hline Lhyn Llagi & 1958.01 .01 & 5.52 & & 22.75 & & & 0.12 & 0.95 & 0.00 & 2.38 & 3.65 & 0.13 & 0.50 & 0.53 \\
\hline Llyn Llsgi & $1999-01-01$ & 5.82 & & 27.78 & & & 0.12 & 7.48 & 0.00 & 2.38 & 4.08 & 0.18 & 0.98 & 0.68 \\
\hline Scost Trn & 1993-01-01 & 5.01 & & 31.50 & & & 0.19 & 5.90 & 0.00 & 3.10 & 3.45 & 0.23 & 0.57 & 0.53 \\
\hline Scost Tarn & $1992-01-01$ & 5.02 & & 33.05 & & & 0.27 & 6.13 & 0.00 & 2.93 & 3.50 & 0.25 & 0.62 & 0.56 \\
\hline Scost Tern & $1958.01 \cdot 01$ & 5.02 & & 31.75 & & & 0.20 & 0.18 & 0.00 & 288 & 3.43 & 0.27 & 0.57 & 0.50 \\
\hline Llyn Llagi & $2008.01-01$ & 5.81 & & 25.00 & & & 0.11 & 5.53 & 0.01 & 1.96 & 3.10 & 0.13 & 0.88 & 0.48 \\
\hline Llyn Llagi & $2005 \cdot 01 \cdot 01$ & 5.75 & & 27.00 & & & 0.06 & 8.40 & 0.01 & 1.93 & 3.40 & 0.11 & 0.88 & 0.49 \\
\hline W indermere & $1994.01 \cdot 01$ & 7.12 & & 87.30 & 10.87 & 0.00 & 0.50 & 7.57 & 0.04 & 5.28 & 4.51 & 0.53 & 5.74 & 0.91 \\
\hline Scost Trrn & $1996.01-01$ & 5.03 & & 35.50 & & & 0.36 & 0.38 & 0.00 & 2.78 & 3.70 & 0.32 & 0.64 & 0.60 \\
\hline Llyn Llagi & $1990.01-01$ & 6.20 & & 48.26 & & & 0.18 & 10.73 & 0.03 & 3.18 & 5.66 & 0.38 & 1.49 & 0.76 \\
\hline Scost Trrn & 1994.01.01 & 5.09 & & 31.50 & & & 0.21 & 5.03 & 0.00 & 3.00 & 3.33 & 0.35 & 0.50 & 0.50 \\
\hline W indermere & $2004.01-01$ & 7.41 & & 51.0 & 10.88 & 0.01 & 0.40 & 0,03 & 0.00 & 3., & 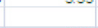 & . & 0.0 & \\
\hline Es thwsite & 2003.01-01 & 7.58 & & 101.25 & 10.71 & 0.03 & 0.38 & 9.02 & 0.01 & 0.67 & 0.46 & 0.90 & 11.12 & 1. 44 \\
\hline Esthwsite & $2000-01-01$ & 7.58 & & 99.28 & 10.72 & 0.04 & 0.32 & 10.09 & 0.05 & 5.88 & 5.78 & 0.88 & 9.70 & 1. 30 \\
\hline Scost Trrn & $2003-01-01$ & 5.22 & & 26.50 & & & 0.22 & 4.78 & 0.00 & 2.48 & 283 & 0.24 & 0.54 & 0.49 \\
\hline Esthwaite & $1994.01-01$ & 7.27 & & 112.83 & 10.38 & 0.08 & 0.43 & 10.21 & 0.00 & 8.88 & 5.83 & 0.89 & 9.84 & 1.30 \\
\hline Scost Trn & 1959-01-01 & 5.07 & & 30.75 & & & 0.25 & 0.03 & 0.00 & 2.53 & 3.38 & 0.22 & 0.52 & 0.50 \\
\hline S cost Tarn & 1950-01-01 & 4.91 & & 44.08 & & & 0.27 & 9.45 & 0.00 & 3.20 & 5.05 & 0.38 & 0.82 & 0.73 \\
\hline Llyn Llagi & 1992.01 .01 & 5.17 & & 3008 & & & 0.19 & 0.43 & 0.01 & 2.90 & 3.70 & 0.15 & 0.90 & 0.53 \\
\hline Llyn Llagi & $1988.01-01$ & E. 40 & & 36.76 & & & 0.18 & 7.30 & 0.03 & 2.93 & 4.08 & 0.47 & 1.08 & 0.80 \\
\hline Llyn Llagi & 1993-01-01 & 5.28 & & 28.30 & & & 0.11 & 5.88 & 0.02 & 3.15 & 3.43 & 0.15 & 1.00 & 0.53 \\
\hline S coot Tern & 1997-01-01 & 8.07 & & 32.26 & & & 0.28 & 0.18 & 0.00 & 2.88 & 3.53 & 0.26 & 0.48 & 0.80 \\
\hline Scost Tarn & $2006-01-01$ & 5.18 & & 27.00 & & & 0.19 & 4.70 & 0.00 & 2.37 & 265 & 0.20 & 0.44 & 0.43 \\
\hline Windermere & $2002-01-01$ & 7.44 & & 63.35 & 10.88 & 0.01 & 0.37 & 8.55 & 0.00 & 0.41 & 4.20 & 0.47 & 5.73 & 0.91 \\
\hline Scost Trn & $2005-01-01$ & 5.12 & & 33.00 & & & 0.20 & 8.35 & 0.00 & 2.23 & 3.40 & 0.22 & 0.52 & 0.52 \\
\hline Llyn Llagi & $2000-01-01$ & 5.69 & & 28.00 & & & 0.05 & 5.10 & 0.00 & 1.98 & 3.03 & 0.13 & 0.75 & 0.50 \\
\hline Esthwaite & $1900 \cdot 01-01$ & 7.83 & & 104.12 & 10.33 & 0.02 & 0.44 & 10.84 & 0.01 & 8.13 & 8.40 & 0.97 & 10.19 & 1. 37 \\
\hline W indermere & $1996-01-01$ & 7.18 & & 89.91 & 9.98 & 0.02 & 0.38 & 8.10 & 0.03 & 5.10 & 4.75 & 0.80 & 5.88 & 0.98 \\
\hline Llyn Llagi & $1996.01 \cdot 01$ & 5.00 & & 32,00 & & & 0.11 & 7.23 & 0.00 & 280 & 4.10 & 0.15 & 1.08 & 0.58 \\
\hline Llyn Llagi & 1991.01.01 & 5.16 & & 39.23 & & & 0.11 & 8.05 & 0.01 & 3.35 & 4.63 & 0.19 & 215 & 0.06 \\
\hline Old Lodge & $2003.01 \cdot 01$ & 5.13 & & 92.75 & & & 0.11 & 18.44 & 0.01 & 9.78 & 10.47 & 0.91 & 3.07 & 1.06 \\
\hline Old Lodge & $1991.01 \cdot 01$ & 4.58 & & 118.88 & & & 0.08 & 21.50 & 0.00 & 14.29 & 10.77 & 0.79 & 3.24 & 1.84 \\
\hline Nant Teyrn & $2002.01 \cdot 01$ & 5.38 & & 2443 & & 0.07 & 0.10 & 0.08 & 0.00 & 0.70 & 3.47 & 0.19 & 0.07 & 0.45 \\
\hline
\end{tabular}


Appendix 2. Continued.

\begin{tabular}{|c|c|c|c|c|c|c|c|c|c|c|c|c|c|c|c|}
\hline $\begin{array}{l}\text { SITENAME } \\
\text { Nant Teyrn }\end{array}$ & $\begin{array}{l}\text { SDATE } \\
\text { 2007-01-01 }\end{array}$ & 5.41 & TS8 & 1.69 & $\begin{array}{r}\text { Conductivity } \\
22.70\end{array}$ & & Ammonium & Nitrate & Chloride & $\begin{array}{r}\text { Phos phate } \\
0.01\end{array}$ & $\begin{array}{l}\text { Sulphate } \\
200\end{array}$ & Sodium & Potass ium & Calcium & Magnes ium \\
\hline Bimie Burn & $1994-01-01$ & 6.69 & & & 63.07 & & 0.02 & 0.10 & 7.99 & 9 $\quad 0.01$ & 211 & 6.89 & 0.68 & 5.08 & 1.56 \\
\hline Bimie Burn & $1997-01-01$ & 6.87 & & & 60.68 & & 0.03 & 0.14 & 8.63 & 0.01 & 1.82 & 6.81 & 0.48 & 4.69 & 1.44 \\
\hline Bimie Burn & 2001-01-01 & 6.80 & & & 70.92 & & 0.01 & 0.14 & 7.28 & 0.01 & 1.79 & 8.20 & 0.47 & 4. 32 & 1.36 \\
\hline Old Lodge & $1999-01-01$ & 4.83 & & & 90.75 & & & 0.09 & 18.50 & 0.00 & 8.56 & 9.67 & 0.60 & 2.76 & 1.54 \\
\hline Trout Beck & $2005-01-01$ & 7.31 & & 2.81 & 80.13 & & 0.03 & 0.10 & 3.98 & 0.00 & 1.31 & 260 & 0.34 & 12. 16 & 0.96 \\
\hline Nant Teyrn & 2001-01-01 & 5.44 & & & 19.51 & & 0.03 & 0.12 & 4.16 & 0.00 & 0.67 & 259 & 0.18 & 0.59 & 0.34 \\
\hline Trout Beck & $1995-01-01$ & 6.09 & & & 86.63 & & 0.02 & 0.15 & 4.18 & 0.00 & 1.98 & 287 & 0.37 & 13.29 & 1.13 \\
\hline Nant Teyrn & 2003-01-01 & 5.56 & & & 26.63 & & 0.04 & 0.10 & 5.06 & 0.01 & 0.71 & 286 & 0.21 & 0.63 & 0.38 \\
\hline Nant Teyrn & $2004-01-01$ & 5.56 & & & 23.94 & & 0.03 & 0.09 & 4.68 & 0.02 & 0.66 & 264 & 0.17 & 0.56 & 0.35 \\
\hline Nant Teyrn & $2005-01-01$ & 5.41 & & & 25.80 & & 0.04 & 0.10 & 5.19 & 0.01 & 0.69 & 2.83 & 0.19 & 0.57 & 0.37 \\
\hline Bimie Burn & $2006-01-01$ & 7.05 & & & 66.52 & 11.22 & 0.02 & 0.20 & 0.85 & 0.01 & 1.86 & 0.38 & 0.48 & 4.35 & 1.36 \\
\hline Bimie Burn & 2007-01-01 & 7.01 & & & 63.13 & & 0.02 & 0.19 & 6.37 & 0.01 & 1.52 & 6.67 & 0.44 & 4.28 & 1.34 \\
\hline Trout Beck & 1994-01-01 & 6.09 & & & 75.57 & & 0.01 & 0.11 & 4.34 & 0.00 & 1.56 & 2.49 & 0.34 & 10.89 & 0.83 \\
\hline Nant Teyrn & $1999-01-01$ & 4.98 & & & 20.97 & & 0.02 & 0.15 & 5.17 & 0.00 & 0.68 & 2.86 & 0.20 & 0.58 & 0.37 \\
\hline Old Lodge & $2005-01-01$ & 5.16 & & & 9200 & & & 0.10 & 19.52 & 0.00 & 7.64 & 10.23 & 0.86 & 2.85 & 1.57 \\
\hline Old Lodge & $1988-01-01$ & 4.52 & & & 77.17 & & & & 20.17 & 0.01 & 9.30 & 10.43 & 0.46 & 2.76 & 1.68 \\
\hline Old Lodge & 1993-01-01 & 4.60 & & & 91.83 & & & 0.10 & 17.40 & 0.00 & 11.02 & 9.46 & 0.74 & 2.71 & 1.49 \\
\hline Old Lodge & $1994-01-01$ & 4.66 & & & 87.50 & & & 0.06 & 17.39 & 0.00 & 8.87 & 9.23 & 0.68 & 2.44 & 1.34 \\
\hline Bush & $1995-01-01$ & 7.93 & & 7.40 & 309.25 & 10.85 & 0.13 & 1.93 & 24.99 & 0.09 & 15.57 & & & & \\
\hline Old Lodge & $1996-01-01$ & 4.60 & & & 108.33 & & & 0.14 & 19.51 & 0.00 & 12.10 & 10.68 & 1.02 & 3.00 & 1.78 \\
\hline Bimie Burn & 2005-01-01 & 7.01 & & & 66.89 & 10.49 & 0.02 & 0.17 & 6.80 & 0.01 & 1.61 & 6.22 & 0.41 & 4.22 & 1.34 \\
\hline Trout Beck & $2000-01-01$ & 6.78 & & 1.94 & 69.44 & & 0.01 & 0.08 & 3.50 & 0.00 & 1.07 & 2.74 & 0.28 & 10.20 & 0.85 \\
\hline Nant Teyrn & 2006-01-01 & 5.48 & & 1.50 & 22.49 & & & & & 0.01 & 1.87 & & & & \\
\hline Trout Beck & $2006-01-01$ & 7.15 & & 1.63 & 76.22 & & 0.02 & 0.09 & 3.60 & 0.00 & 1.33 & 246 & 0.30 & 11.40 & 0.91 \\
\hline Bimie Burn & $1998-01-01$ & 6.95 & & & 46.00 & & 0.04 & 0.16 & 8.45 & 0.03 & 1.77 & 6.27 & 0.49 & 3.65 & 1.25 \\
\hline Trout Beck & $1993-01-01$ & 6.93 & & & 44.48 & & 0.00 & 0.13 & 4.93 & 0.00 & 1.65 & 2.80 & 0.35 & 10.65 & 0.94 \\
\hline Bimie Burn & $1999-01-01$ & 7.05 & & & 51.65 & & 0.02 & 0.15 & 8.07 & 0.00 & 1.78 & 6.56 & 0.49 & 4.23 & 1.37 \\
\hline Bush & 1998-01-01 & 7.83 & & 7.14 & 314.12 & 11.59 & 0.10 & 3. 19 & 25.82 & 0.10 & 14.57 & & & & \\
\hline Old Lodge & 1997-01-01 & 4.57 & & & 102.17 & & & 0.12 & 19.43 & 0.00 & 10.05 & 9.86 & 0.85 & 2.65 & 1.55 \\
\hline Bimie Burn & $1993-01-01$ & 6.58 & & & 65.71 & & 0.02 & 0.11 & 7.86 & 0.01 & 1.85 & 6.10 & 0.70 & 3.94 & 1.29 \\
\hline Bimie Burn & 2003-01-01 & 6.94 & & & 76.98 & & 0.01 & 0.17 & 7.59 & 0.01 & 1.97 & 6.67 & 0.45 & 5.12 & 1.51 \\
\hline Bush & $1997-01-01$ & 8.02 & & 6.71 & 318.28 & 11.60 & 0.07 & 2.77 & 27.05 & 0.10 & 17.11 & & & & \\
\hline Old Lodge & $2006-01-01$ & 5.02 & & & 113.55 & & & 0.11 & 26.33 & 0.00 & 820 & 1222 & 1.04 & 287 & 1.63 \\
\hline Bimie Burn & 2004-01-01 & 6.72 & & & 63.04 & & 0.01 & 0.14 & 7.12 & 0.00 & 1.75 & 0.15 & 0.35 & 3.89 & 1.25 \\
\hline Old Lodge & $1992-01-01$ & 4.54 & & & 10242 & & & 0.10 & 19.74 & 0.00 & 11.29 & 9.93 & 0.78 & 2.84 & 1.58 \\
\hline Old Lodge & 2001-01-01 & 5.13 & & & 84.18 & & & 0.10 & 17.08 & 0.01 & 6.66 & 9.45 & 0.76 & 2.66 & 1.35 \\
\hline Trout Beck & $2007-01-01$ & 6.88 & & & 74.84 & & 0.02 & 0.08 & 4.27 & 0.00 & 1.05 & 2.83 & 0.29 & 10.97 & 0.87 \\
\hline Bimie Burn & $1996-01-01$ & 6.77 & & & 58.12 & & 0.01 & 0.16 & 8.33 & 0.00 & 2.03 & 6.75 & 0.47 & 4.36 & 1.47 \\
\hline Bimie Burn & $2000-01-01$ & 6.69 & & & 53.36 & & 0.02 & 0.11 & 7.25 & 0.00 & 1.61 & 5.95 & 0.45 & 3.81 & 1.25 \\
\hline Trout Beck & $1996-01-01$ & 5.95 & & & 89.21 & & 0.03 & 0.24 & 4.53 & 0.00 & 1.79 & 3.06 & 0.38 & 1234 & 1.11 \\
\hline Bimie Burn & $2002-01-01$ & 6.73 & & & 58.83 & & 0.01 & 0.12 & 6.73 & 0.01 & 1.65 & 5.68 & 0.35 & 3.91 & 1.20 \\
\hline Old Lodge & $1990-01-01$ & 4.45 & & & 12851 & & & & 23.85 & 0.01 & 17. 13 & 12.79 & 0.73 & 3.94 & 220 \\
\hline Old Lodge & $1995-01-01$ & 4.62 & & & 112.67 & & & 0.09 & 20.52 & 0.00 & 11.64 & 11.38 & 0.75 & 3. 10 & 1.78 \\
\hline Trout Beck & 2004-01-01 & 6.78 & & 3.73 & 64.69 & & 0.03 & 0.07 & 3.44 & 0.00 & 1.07 & 2.38 & 0.30 & 10.26 & 0.80 \\
\hline Trout Beck & $2001-01-01$ & 7.18 & & 2.36 & 78.65 & & 0.03 & 0.08 & 3.30 & 0.00 & 1.25 & 284 & 0.33 & 11.76 & 0.96 \\
\hline Old Lodge & $2000-01-01$ & 4.90 & & & 84.25 & & & 0.11 & 17.87 & 0.00 & 8.07 & 9.36 & 0.80 & 2.80 & 1.75 \\
\hline Old Lodge & 2004-01-01 & 5.00 & & & 97.08 & & & 0.11 & 19.80 & 0.00 & 975 & 9.54 & 0.80 & 295 & 1.59 \\
\hline Old Lodge & $1989-01-01$ & 4.55 & & & 104.36 & & & & 24.06 & 0.01 & 15.00 & 12.49 & 0.76 & 3.76 & 2.14 \\
\hline Nant Teyrn & $2000-01-01$ & 5.25 & & & 19.79 & & 0.02 & 0.12 & 426 & 0.00 & 0.67 & 247 & 0.18 & 0.52 & 0.32 \\
\hline Trout Beck & $1997-01-01$ & 6.11 & & 2.29 & 84.07 & & 0.02 & 0.16 & 4. 17 & 0.00 & 1.40 & 2.87 & 0.34 & 12.36 & 1.05 \\
\hline Trout Beck & $1999-01-01$ & 7.03 & & 1.63 & 75.42 & & 0.02 & 0.09 & 4.44 & 0.00 & 1.21 & 2.90 & 0.35 & 10.88 & 0.93 \\
\hline Trout Beck & 1998-01-01 & 6.14 & & 1.61 & 61.33 & & 0.02 & 0.11 & 4. 18 & 0.00 & 1.14 & 2.59 & 0.27 & 9.07 & 0.79 \\
\hline Bimie Burn & 1995-01-01 & 6.81 & & & 60.61 & & 0.02 & 0.10 & 7.69 & 0.01 & 207 & 6.71 & 0.53 & 4.55 & 1.45 \\
\hline \multirow[t]{2}{*}{ Esthwaite } & $1997-01-01$ & 7.45 & & & 108.33 & 10.41 & 0.05 & 0.66 & $\quad 11.40$ & 0.01 & 7.25 & 6.17 & 1.25 & 9.90 & 1.32 \\
\hline & & $\mathrm{pH}$ & TSS & & Conductivity D & 00 & Ammonium & Nitrate & Chloride & Phosphate & Sulphate & Sodium & Potas sium & Calcium & Magnesium \\
\hline 1988-2007 & mean & 608 & & 3.46 & 71.62 & 10.65 & $\quad 0.03$ & 0.31 & 9.52 & $2 \quad 0.01$ & 4.33 & $\begin{array}{l}3.25 \\
\end{array}$ & 0.46 & 4.32 & $2 \quad 0.99$ \\
\hline NI and Sco & min & 4.45 & & 1.43 & 14.50 & 9.91 & 0.00 & 0.05 & 3.30 & 0.00 & 0.41 & 2.38 & 0.11 & 0.44 & 0.32 \\
\hline lakes and & & 808 & & 7.40 & 329.24 & 100 & 0.14 & 3.19 & 27.05 & 0.10 & 17.13 & 6 & (2) & 15.48 & 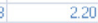 \\
\hline
\end{tabular}




\section{Appendix 3.}

\begin{tabular}{|c|c|c|c|c|c|c|c|c|c|c|c|c|c|c|c|c|c|c|c|c|c|c|c|c|c|}
\hline Conductivity & UK & Jav and & Foster I & Lillev $\mathrm{S}$ & Smith & Demers $L$ & Lvons a $\mathrm{A}$ & Arrign $A$ & Arrign & Broqu $\mathrm{R}$ & Reviol a 1 & a Trouilh & Trouill & Troschel ( G & Garcia-ı & Rallo a $\mathrm{I}$ & Nardi e $\mathrm{S}$ & Scalici Re & Renai et al. & Barba $\mathrm{B}$ & Bruscc $\mathrm{P}$ & Favarc & Trožic G & Gottste & e This stu $n$ \\
\hline Minimum & 142.83 & & 93 & 60 & 93 & 37 & & & 310 & & 6 & $5 \quad 110$ & 101 & 225 & 245 & 144 & 197 & & 141 & 271 & 245.4 & & 180 & & \\
\hline Mean & 515.74 & & & & & 427 & & 160 & 335 & 618 & 40 & 138.5 & 233 & 272 & 372 & 376 & 540.8 & & 350 & 424 & 373.3 & 225 & 225.4 & & \\
\hline Maximum & 1289.04 & & 507 & 390 & 507 & 772 & & & 385 & & 66 & 161 & 596 & 345 & 499 & 607 & 945 & & 514 & 552 & 466 & & 310 & & \\
\hline Potassium & UK & 1 & 2 & 3 & 4 & 5 & 6 & 7 & 8 & 9 & 10 & 11 & 12 & 13 & 14 & 15 & 16 & 17 & 18 & 19 & 20 & 21 & 22 & & $3 \mathrm{NRH}$ \\
\hline Minimum & 0.11 & & 0.3 & 0.8 & 0.8 & & & & & & & 2 & 0.1 & & 0 & 0.01 & & & & & & & & & 0.067 \\
\hline Mean & 0.46 & & & & & & & & & & & 2.9 & 2.8 & & 2.44 & 1.7 & & & & & & & & & 2.062 \\
\hline Maximum & 1.25 & & 2.5 & 3.9 & 2.5 & & & & & & & 4.2 & 9.6 & & 5.4 & 12.2 & & & & & & & & & 5.784 \\
\hline Magnesium & UK & 1 & 2 & 3 & 4 & 5 & 6 & 7 & 8 & 9 & 10 & 11 & 12 & 13 & 14 & 15 & 16 & 17 & 18 & 19 & 20 & 21 & 22 & & $3 \mathrm{NRH}$ \\
\hline Minimum & 0.32 & & 1.6 & 1.8 & 3 & & & & 5 & & 0.9 & & 0 & & 4.31 & 2.3 & & & & & & & & & 1.381 \\
\hline Mean & 0.99 & & & & & & & 8 & 8.5 & 12.7 & 1.2 & & 4.3 & & 6.84 & 6 & & & & & & 12.7 & & & 6.976 \\
\hline Maximum & 2.20 & & 10.5 & 12.3 & 10.5 & & & & 11.5 & & 2.3 & & 6.9 & & 9.37 & 11.4 & & & & & & & & & 28.779 \\
\hline Ammonium & UK & 1 & 2 & 3 & 4 & 5 & 6 & 7 & 8 & 9 & 10 & $\quad 11$ & 12 & 13 & 14 & 15 & 16 & 17 & 18 & 19 & 20 & 21 & 22 & & $3 \mathrm{NRH}$ \\
\hline Minimum & 0.02 & & 0.01 & & 0.01 & & & 0 & 0 & 0 & 0.028 & 0.002 & & 0.001 & 0 & 0 & & 0 & & & & 0.01 & & & 0.001 \\
\hline Mean & 0.42 & & & & & & & 0 & 0.06 & 0.06 & 0.055 & 0.039 & & 0.03 & 0.17 & 0 & & 0.25 & 0.427 & & & 0.12 & & 0.04 & 0.058 \\
\hline Maximum & 5.14 & & 0.74 & & 0.05 & & 0.004 & 0 & 0 & 0.1 & 0.148 & 0.114 & & 0.07 & 0.36 & 0 & & 0.7 & & & & 0.73 & & & 0.305 \\
\hline Calcium & UK & 1 & 2 & 3 & 4 & 5 & 6 & 7 & 8 & 9 & 10 & 11 & 12 & 13 & 14 & 15 & 16 & 17 & 18 & 19 & 20 & 21 & 22 & & $3 \mathrm{NRH}$ \\
\hline Minimum & 0.44 & & 6.5 & 6.1 & 7.9 & & & & 52 & & 1.2 & 40 & 1 & & 29.79 & 15 & & & 28.3 & 43.0 & 47.1 & & & & 7.437 \\
\hline Mean & 4.32 & & & & & & & 40 & 66 & 125 & 7.67 & 60.75 & 27.2 & & 42.02 & 40 & & & 63.5 & 71.7 & 69 & 28.4 & & & 24.557 \\
\hline Maximum & 15.48 & & 94.7 & 66.8 & 94.7 & & & & 78 & & 8.5 & 100 & 99.3 & & 54.25 & 66 & & & 94.5 & 100.8 & 92.2 & & & & 58.306 \\
\hline Sodium & UK & 1 & 2 & 3 & 4 & 5 & 6 & 7 & 8 & 9 & 10 & 11 & 12 & 13 & 14 & 15 & 16 & 17 & 18 & 19 & 20 & 21 & 22 & & $3 \mathrm{NRH}$ \\
\hline Minimum & 2.38 & & 5.6 & 7 & 5.7 & & & & & & & 6.7 & 2.5 & & 6.81 & 4.3 & & & & & & & & & 3.103 \\
\hline Mean & 5.25 & & & & & & & & & & & 9.95 & 6.91 & & 10.34 & 9.4 & & & & & & & & & 15.779 \\
\hline Maximum & 12.79 & & 13 & 12.1 & 13 & & & & & & & 14.8 & 17 & & 13.87 & 17.5 & & & & & & & & & 50.300 \\
\hline Chloride & UK & 1 & 2 & 3 & 4 & 5 & 6 & 7 & 8 & 9 & 10 & 11 & 12 & 13 & 14 & 15 & 16 & 17 & 18 & 19 & 20 & 21 & 22 & & $3 \mathrm{NRH}$ \\
\hline Minimum & 13.35 & & 0.6 & & 8 & & & & 14 & & 7.2 & 11.3 & 5.7 & 10 & 8.9 & 5 & & & & & & & & & 2.951 \\
\hline Mean & 54.49 & & & & & & & 8 & 18 & & 7.67 & 16.06 & 17.7 & 33 & 14.3 & 13 & & & & & & 11.2 & & & 27.363 \\
\hline Maximum & 185.33 & & 37.3 & & 23 & & & & 24 & & 8.1 & 27.6 & 42.7 & 60 & 19.7 & 25 & & & & & & & & & 85.218 \\
\hline Nitrate & UK & 1 & 2 & 3 & 4 & 5 & 6 & 7 & 8 & 9 & 10 & 11 & 12 & 13 & 14 & 15 & 16 & 17 & 18 & 19 & 20 & 21 & 22 & & $3 \mathrm{NRH}$ \\
\hline Minimum & 2.68 & & 0.6 & & 0.6 & & & & 0.6 & & 0 & 9.88 & 3.7 & 0.05 & 0.6 & 0.01 & & 0.01 & 0 & 0 & 1.7 & & & & 0.107 \\
\hline Mean & 21.57 & & & & & & & 1.6 & 0.6 & 25.8 & 3.67 & 16.01 & 17.7 & 2.8 & 3.12 & 2.7 & & 0.11 & 4.3 & 0.943 & 3.578 & 7.7 & & & 7.362 \\
\hline Maximum & 47.64 & & 4.2 & & 4.2 & & & & 0.8 & & 4.5 & 27.47 & 57.2 & 5 & 5.64 & 9.1 & & 0.78 & 8 & 2.5 & 5 & & & & 20.897 \\
\hline Sulphate & UK & 1 & 2 & 3 & 4 & 5 & 6 & 7 & 8 & 9 & 10 & 11 & 12 & 13 & 14 & 15 & 16 & 17 & 18 & 19 & 20 & 21 & 22 & & $3 \mathrm{NRH}$ \\
\hline Minimum & 0.41 & & 8.8 & & 8.4 & & & & 6 & & 0 & 5.2 & 1.7 & & 17.1 & 7 & & & & & & & & & 0.881 \\
\hline Mean & 4.33 & & & & & & & 2 & 7 & & 0 & 8.08 & 9.33 & & 30.8 & 29 & & & & & & 76.6 & & & 42.411 \\
\hline Maximum & 17.13 & & 23.6 & & 23.6 & & & & 8 & & 0 & 11.51 & 26.2 & & 44.5 & 87 & & & & & & & & & 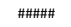 \\
\hline Phosphate & UK & 1 & 2 & 3 & 4 & 5 & 6 & 7 & 8 & 9 & 10 & 11 & 12 & 13 & 14 & 15 & 16 & 17 & 18 & 19 & 20 & 21 & 22 & 23 & $3 \mathrm{NRH}$ \\
\hline Minimum & 0.01 & & 0.01 & & 0 & & & & 0 & & 0.01 & & & & & 0.01 & & 0.01 & & & & & & & 0 \\
\hline Mean & 0.20 & & & & & & & 0 & 0 & 0.1 & 0.02 & & & & & 0.07 & & 0.05 & & & & 0.56 & & 0.02 & 0.029 \\
\hline Maximum & 0.72 & & 0.236 & & 0.22 & & & & 0 & & 0.03 & & & & & 0.39 & & 0.1 & & & & & & & 0.11 \\
\hline DO & UK & 1 & 2 & 3 & 4 & 5 & 6 & 7 & 8 & 9 & 10 & 11 & 12 & 13 & 14 & 15 & 16 & 17 & 18 & 19 & 20 & 21 & 22 & & $3 \mathrm{NRH}$ \\
\hline Minimum & 6.01 & & 9.5 & & & & 4.4 & & & & 8.1 & 7.32 & 4.93 & & & & 4.5 & 3.4 & 6.57 & 5.2 & 6.6 & & 9.7 & & 4.490 \\
\hline Mean & 10.13 & & & & & & & & & 8.58 & 10.67 & 8.115 & 7.84 & & & & 8.56 & 4.67 & 9.16 & 7.437 & 7.778 & 8.30 & 11.29 & & 10.275 \\
\hline Maximum & 12.20 & & 11.6 & & & & & & & & 12.2 & 10.35 & 12.5 & & & & 15.7 & 6.1 & 12.21 & 8.7 & 8.8 & & 13.1 & & 19.400 \\
\hline & UK & 1 & 2 & 3 & 4 & 5 & 6 & 7 & 8 & 9 & 10 & 11 & 12 & 13 & 14 & 15 & 16 & 17 & 18 & 19 & 20 & 21 & 22 & & $3 \mathrm{NRH}$ \\
\hline Minimum & 7.09 & 6 & 7.27 & 6.8 & 7.1 & 7.24 & & & 8.4 & & 6.5 & 6.51 & 6.23 & 6.8 & 7.95 & 7.6 & 7.6 & 7 & 7.56 & 7.6 & 7.7 & & & & \\
\hline Mean & 7.79 & & & & & 7.84 & & 7.6 & 8.4 & 7.45 & 7.33 & 7.025 & 7.3 & 7.4 & 8.2 & 8.1 & 8.22 & & 8.2 & 8.088 & 8.089 & 7.9 & & & \\
\hline Maximum & 8.38 & 9.2 & 8.64 & 8.5 & 8.6 & 8.54 & & & 8.4 & & 7.6 & 7.48 & 8.69 & 7.9 & 8.45 & 8.6 & 8.8 & 8 & 8.73 & 8.3 & 8.6 & & & & \\
\hline & UK & 1 & 2 & 3 & 4 & 5 & 6 & 7 & 8 & 9 & 10 & 11 & 12 & 13 & 14 & 15 & 16 & 17 & 18 & 19 & 20 & 21 & 22 & & $3 \mathrm{NRH}$ \\
\hline Minimum & 2.72 & & 7.5 & & & & & & & & & 2.8 & 0.2 & & & & & & & & & & & & \\
\hline Mean & 18.03 & & & & & & & & & 7 & & 11.45 & 16.9 & & & & & & & & & & & & \\
\hline Maximum & 85.70 & & 33.6 & & & & & & & & & 28 & 489 & & & & & & & & & & & & \\
\hline
\end{tabular}


N.R. Haddaway et al.: Knowl. Managt. Aquatic Ecosyst. (2015) 416, 01

Appendix 3. Continued.

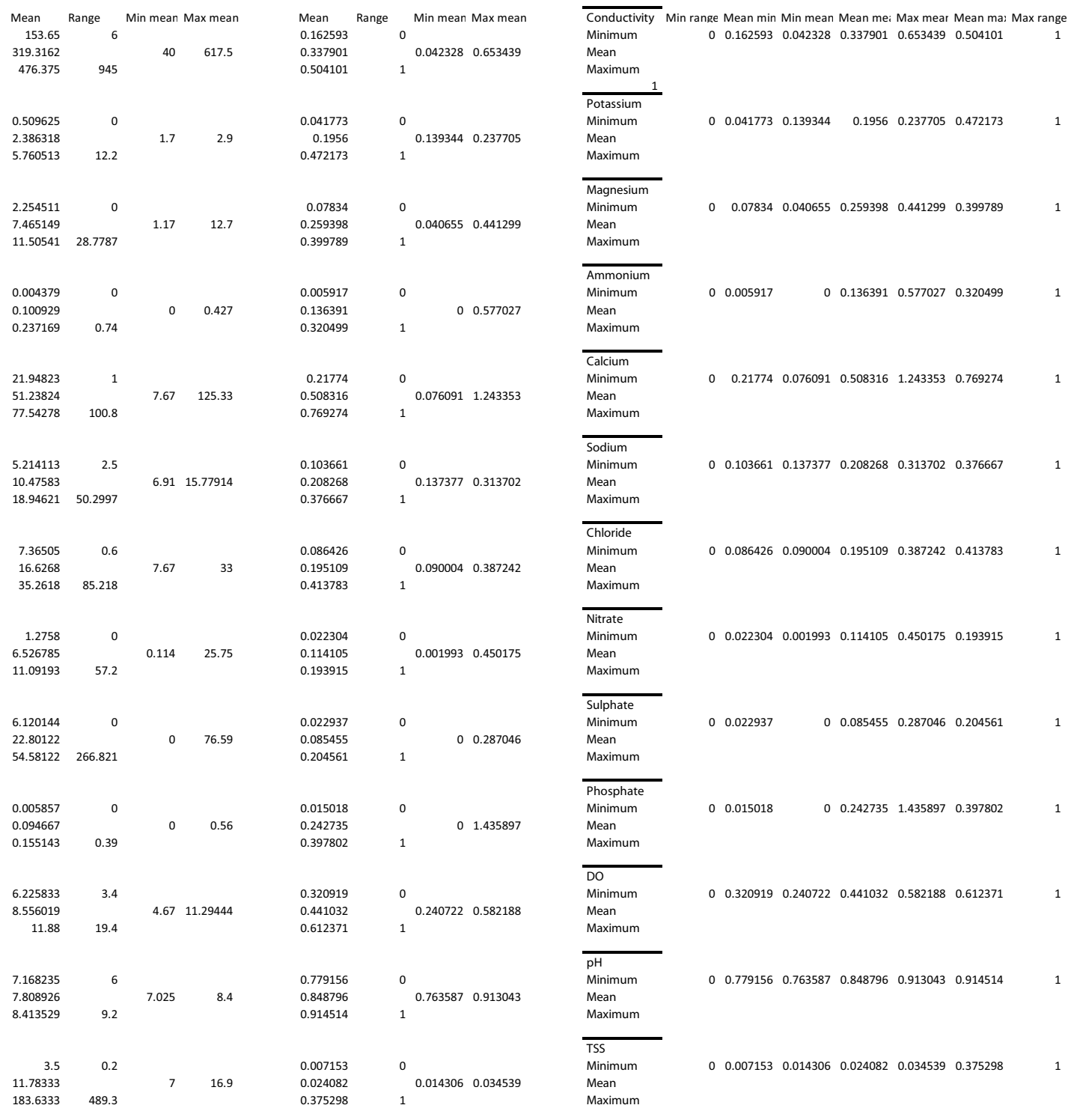

